\title{
Impact of pulse time uncertainty on synchronous average: Statistical analysis and relevance to rotating machinery diagnosis
}

\author{
V. Camerini ${ }^{\mathrm{a}, \mathrm{b}, *}$, G. Coppotelli ${ }^{\mathrm{a}}$, S. Bendisch ${ }^{\mathrm{b}}$, D. Kiehn ${ }^{\mathrm{c}}$ \\ a Department of Mechanical and Aerospace Engineering, University of Rome “La Sapienza", 00184 Rome, Italy \\ b Airbus Helicopters Germany, 86609 Donauwörth, Germany \\ ${ }^{\mathrm{c}}$ German Aerospace Center (DLR), Institute of Flight Systems, 38108 Braunschweig, Germany
}

\section{A R T I C L E I N F O}

\section{Article history:}

Received 15 September 2018

Received in revised form 27 January 2019

Accepted 11 April 2019

\section{Keywords:}

Synchronous average

Gearbox

Helicopter

HUMS

Cyclostationarity

Statistical analysis

Signal processing

Estimation uncertainty

\begin{abstract}
A B S T R A C T
Time synchronous averaging for the extraction of periodic waveforms is a rather common processing method for rotating machinery diagnosis. By synchronizing the signal to the rotational angle of the component of interest, e.g. by using a keyphasor reference signal, it is possible to perform the averaging in the angular domain, thus obtaining an anglesynchronous signal. Jittering of the reference signal affects the quality of the synchronous averaging process, resulting in attenuation and uncertain estimation of the extracted synchronous signal, especially in the high frequency band. In this paper, the effects of random uncertainty in the pulse arrival times of the reference signal on the synchronous averaging method are studied, with the objective of assessing the relevance of such a jitter error to the extracted waveform and the indicators derived for monitoring purposes. First, a unified framework for the computed order tracking method is presented, and then a model linking the statistics of the random jitter to the statistics of the waveform extracted through synchronous averaging in angle domain is developed. The theoretical model connects the random jitter distribution, the number of averaged periods and the ratio of the period of interest to the reference trigger period, to the distribution of the amplitudes of the synchronous frequency components in the synchronously averaged signal. Approximate analytical solutions are derived for cases of interest, allowing the prediction of the attenuation bias and variability of the extracted components amplitudes. The model is first verified against numerical simulations in order to assess consistency, and then parametric studies are presented. Experimental validation is performed on both an experimental and an operational data sets involving respectively a helicopter gearbox and a helicopter fleet.
\end{abstract}

(c) 2019 Elsevier Ltd. All rights reserved.

\section{Introduction}

In the last decades rotating machinery vibration signals have been successfully modelled as cyclostationary processes [1-5]. Cyclostationarity is a property characterizing stochastic processes whose statistics vary periodically with respect to

\footnotetext{
Abbreviations: HUMS, Health and Usage Monitoring Systems; CI, Condition Indicator; SA, Synchronous Average.

* Corresponding author at: Department of Mechanical and Aerospace Engineering, University of Rome "La Sapienza", 00184 Rome, Italy.

E-mail address: valerio.camerini@uniroma1.it (V. Camerini).
} 
some variable (for rotating machinery, typically time or shaft's angular position). Due to this generality, cyclostationarity property is particularly fit to describe rotating machinery signals [6]. Formally, a first order time-cyclostationary signal (CS1) $y(t)$ is such that its expected value $m_{y}(t)$ is periodic with some period $T$ [6], i.e.:

$$
m_{y}(t) \triangleq E[y(t)]=m_{y}(t+T),
$$

where $E[\cdot]$ is the ensemble average operator. More generally, a (pure) $n$-th order cyclostationary signal (indicated as CSn) has periodic $n$-th order statistical moments (cumulants). Among CSn signals, the class of CS1 and CS2 are of particular importance in machinery diagnostic. Specifically, the CS1 part of the signal carries important information about gears and shafts health, e.g. it is correlated with unbalance of the shafts and with the teeth-meshing process of gears [6] and has been widely used for diagnostic purposes [7-19]. The CS2 part was instead shown to carry information about potential bearing faults [4]. Rotating machinery signals often contain multiple periodicities (i.e. they are composed of different CS components of different period $T$, associated to components rotating at different speeds). They are then classified as poly-cyclostationary (PC) processes. According to [6], this means that the set of their statistical Fourier coefficients is the combination of the statistical Fourier coefficients of all the basic cyclostationary signals composing the poly-cyclostationary signal, i.e. the periodic statistical cumulants have a Fourier series expansion that can be expressed by superposing the fundamental cycles present in the signal. Thus, in a PC signal there exist more CS components with different fundamental periods, allowing most of the rotating machine signals to be covered by simply enlarging the formalism of cyclostationary processes to poly-cyclostationary processes [6].

An accurate estimation of the CS1 component of a signal is key of paramount importance to the diagnostics procedure of rotating machinery for two reasons. First, for its important role in gears and shafts diagnostic as mentioned, and secondly, by subtracting the periodic mean from the signal, the pure CS2 part can be directly analyzed, which is correlated with, e.g., bearing faults. In [6], the operator $P_{A}[y(\cdot)]$ was introduced as the operator that extracts from signal $y(\cdot)$ its components having periodicities in $A$, being $A$ a subset of all the possible periodicities in the signal space to which $y$ belongs. For a CS1 signal, the periodic part coincides with its expected value, and hence the ensemble average of Eq. (1) can be replaced by the operator $P$ that extracts all the periodic components of a signal. This property is the equivalent of ergodicity for a stationary stochastic process, and is thus named cycloergodicity. The $P$ operator can be given either in terms of infinite cycle average or in terms of a Fourier series. In the form of infinite cycle average, the application of the $P$ operator to a CS signal $y(t)$ reads:

$$
P[y(t)]=\lim _{N \rightarrow \infty} \frac{1}{2 N+1} \sum_{i=-N}^{N} y(t+i T)
$$

Eq. (2) is of practical relevance, highlighting the equivalence of cycle averaging with ensemble averaging for cycloergodic signals [6]. Such an equivalence enables the estimation of the periodic mean $m_{y}(t)$ from a single realization of the process of interest. In a way, this presents analogies with the power spectral density (PSD) estimation through segment averaging: the property of cycloergodicity for CS signals is in fact the equivalent of the ergodicity property for stationary signals. Since any measurement process results in a finite-length signal, $m_{y}(t)$ needs in practice to be estimated from a finite set of cycles. Following [6], estimators of $m_{y}(t)$ are referred to as first order cyclostationary tools.

Two interesting cases can be distinguished. In one case, the fundamental cycle of the CS signal is known a priori, e.g. from the knowledge of the kinematic relations in a machine. In the other, such fundamental cycle is unknown and one must resort to blind estimators, as the linear adaptive enhancer (ALE), or the self-adaptive noise canceller (SANC) [20-23]. Generally, blind estimators performance is negatively affected by signal to noise ratio. Moreover, blind filters require a proper parameter tuning which may not be trivial in every case. When the cycle of the signal is known, a popular estimator of $m_{y}(t)$ is the synchronous average (SA) operator, which is also known as Time Synchronous Average (TSA) due to its original formulation in time domain. SA is basically approximating Eq. (2) with a finite number of averages $N$.

Rotating machinery signals are better described as periodic in the angular domain defined by the reference shaft rotation angle (i.e. they are angle-cyclostationary rather than time-cyclostationary). Therefore, they are better analyzed in the angledomain, where the cyclostationary property is preserved. The description of a signal in the angular domain is typically achieved through computed order tracking [24], which consists of resampling the time signal at constant-angle increments based on the information from a reference signal, that might come for example from a pick-up or a tachometer. The reference consists typically of a certain, known number of pulses per revolution of a reference shaft, which can be used as a reference for resampling the signal at constant angular increments. Once the resampled signal $x(\theta)=y(t(\theta))$ is obtained as a function of the reference shaft angle $\theta$ (which can be expressed in shaft revolution units), the periodic mean $m_{x}(\theta)$ can be estimated given the fundamental cycle $\theta$. In absence of a tachometric reference signal, the tacho pulse instants may still be estimated directly from the vibration signal under the condition of very small speed fluctuations, e.g. through demodulation of a gearmesh harmonic as in [25-27].

Only small speed fluctuations allow to preserve first order cyclostationarity in the response signal, since the signal statistics are affected by the different operating speed regimes [28]. For the general case of non-negligible speed fluctuations, solutions based on the concept of fuzzy cyclostationarity [29] were studied e.g. in [17,30]. Practically, the reference pulse time of arrival suffers from a random jitter error arising from the measure limitations, physical imperfections, electrical perturbations or acquisition unit software limitations, whereas estimation errors are introduced when trying to compensate for the speed variation directly through the measured vibration signal. In [24], the authors analyse which factors and assump- 
tions have the greatest effect on the accuracy of the computed order tracking. Since then, keyphasor precision issues and their effect on angular resampling and instantaneous angular speed estimation attracted significant research interest [3133].

A first objective of this paper is to entirely formalizing the angular resampling and the computed order tracking methodology in a solid theoretical framework. Such a formalization offers a simple, unified vision of the problem of approximating the time-angle relationship based on the approximate knowledge of a set of couples of angle and time. Taking advantage of the developed formalization, a comprehensive analysis of the effect the random jitter error affecting the known keyphasor arrival times on the synchronous averaging procedure is then performed, based on the first results presented in [34], with the objective of quantifying the error on the amplitude of the estimated periodic components. The random error has mainly two effects: on one hand, it results in an attenuation bias of the estimated amplitudes of high frequency periodic components; on the other hand, it introduces additional variability of the estimates, possibly hindering relevant information from the periodic amplitudes measurements. With respect to [34], several extensions are presented in this article. First, the developed formalism explicitly allows to consider the speed fluctuations in the mapping to the angular domain; then, the treatment of cyclic averages on cycles which are not multiples of the fundamental keyphasor cycle is considered. The effect of signal measurement noise on the estimates is discussed. Also, the statistical analysis is made more rigorous by making explicit the mixing covariance matrix terms, which improved the results of the analytical approximation originally derived in [34]. Further, more comprehensive numerical and experimental validations are provided, including operational helicopters data.

The remainder of this paper is organised as follows: in Section 2, the angular sampling, computed order tracking and synchronous averaging procedures are introduced; then, the problem of random jitter effect is discussed. In Section 3, the modelling of SA in presence of a random jitter error affecting tacho pulses arrival time is discussed. A new formulation is proposed for studying CS1 signals. An approximated analytical solutions for the distribution of the estimated amplitudes of the periodic components in the extracted signal is then derived from the theoretical model as a function of the extent of pulse time uncertainty, the number of periods used for estimation and the ratio of the period of interest to the reference trigger period. In Section 4, numerical simulations are presented in which the prediction capabilities of the model are verified and the impact of jitter errors on the recovery of CS1 signals through SA method is shown. Experimental verification is performed for two cases in Section 5: one involving a bench test experiment on an Airbus type 1 helicopter gearbox and one involving flight data analysis from a fleet of Airbus type 2 helicopters. Practical implications for the monitoring are also discussed. Finally, conclusions are drawn in Section 6.

\section{Theoretical background}

The content of this section is a summary of known material, mostly based on [24,35-38]. The concept of angular sampling and the methods of computed order tracking and synchronous averaging are introduced along with the adoption of a unified notation, which allows to connect the analysis later developed in the paper with the theoretical framework already present in the literature.

\subsection{Angular sampling and computed order tracking}

Vibration signals recorded from accelerometers are normally sampled at constant time intervals. When analyzing rotating machinery signals, angular sampling is a common strategy adopted to correct for shaft speed fluctuation and obtain a synchronized signal with periodic statistics (i.e. a cyclostationary signal) [33], allowing to exploit cyclostationary tools for diagnostic purposes [39].

Angular sampling may be performed both directly, when proper hardware is available, or in a post-processing step (in that case the procedure is referred to as angular resampling), when the signal is originally acquired using a classical time domain acquisition board. Direct angular sampling requires a data acquisition board with an external clock and a variable anti-aliasing filter, since Nyquist frequency varies with time according to the sampling interval. Such a solution is much more expensive than other systems and commercially less interesting [26]. Further, typical industrial vibration monitoring systems often provide sampling in time domain, hence angular sampling has to be achieved through a post-processing step. Although this leads to decreased accuracy with respect to direct angular sampling, there is an advantage in obtaining the vibration signal both in time and angle domain, since it is often beneficial to have a joint description in order to capture both those features of the signal which are better described as angle-periodic (e.g. impulses generated synchronously with shaft revolutions) and those features which are better described in time domain (e.g. the impulse responses of a linear time invariant system) [40].

The most common method to resample in angle domain was introduced and studied in [24,35] and it is known as computed order tracking. Using a keyphasor reference signal from an optical encoder or a tachometer, providing one or more pulses per revolution, pulse arrival times are obtained and subsequently used to approximate the relationship between time and angular position of the shaft. Alternatively, when no encoder signal is available, the angular position can be estimated from the acceleration signal itself, using e.g. a meshing frequency as in [26]. In the original proposal from [35], the missing angular values are obtained by fitting a second order polynomial to three consecutive pulse arrival times, assuming therefore 
a piecewise constant acceleration of the shaft. Assuming a shaft inertia high enough to prevent backlashing, it is possible to formally define a bijective relationship $\phi: t \rightarrow \theta(t)$ between the time variable $t$ and the generic shaft angular position variable $\theta$ (expressed in shaft revolution units). Such an angle progression relationship might include a predictable evolution $f(t)$, related e.g. to a ramp-up law or constant operation shaft speed, as well as a term $r(t)$ originating from random shaft speed fluctuation, so that $\theta$ can be seen as a random function of the independent, deterministic variable $t$. Maintaining a similar notation as in [6], it is possible to write:

$$
\phi: t \rightarrow \theta(t)=f(t)+r(t)=\int_{-\infty}^{t} \omega(u) d u+\int_{-\infty}^{t} v(u) d u
$$

where $\omega(t)>0$ is the deterministic instantaneous angular speed of the shaft at time t, and $v(t)$ is a random speed fluctuation around $\omega(t)$ such that $|v(t)|<\omega(t)$ for any $t$ in order to preserve bijectivity [6]. An important observation can be when done modelling the rotor system as a second-order system in the torsional degree of freedom. By Laplace-transforming the equations of motion, it is possible to observe that the rotational inertia has the effect of a low-pass filter on the angular speed response to perturbations of the applied moments (torque and resisting aerodynamics/frictional forces), resulting therefore in reasonably band-limited speed fluctuations for high values of the rotor inertia. Therefore, the higher the inertia of the considered rotor, the slower will be the relevant speed fluctuations. Such a filtering effect restricts the effective bandwidth of the random perturbation $v(t)$, allowing to use the encoder signal for reconstruction of the speed (shaft angle) profile without incurring in significant aliasing effects.

The first step of order tracking corresponds to determining a bijective relationship $\hat{\phi}: t \rightarrow \hat{\theta}(t)$ which approximates $\phi$ based on the knowledge of $\theta(t)$ for $t$ belonging to the discrete set of times $\mathbb{v}$ such that the shaft angle position is exactly known, i.e. $\hat{\theta}(t \in \mathbb{T})=\theta(t \in \mathbb{T})$. This step is usually accomplished through interpolation. The set $\mathbb{T}$ is that of the ideal pulse arrival times. In the adopted notation, in order to avoid confusion, the transformation from $t$ to $\hat{\theta}$ through $\hat{\phi}$ is written as $\hat{\theta}=\hat{\phi}(t ; \mathbb{X})$, with $\mathbb{X}$ denoting the set of pulse arrival times used for the estimation of $\hat{\phi}$. The same notation holds for the reverse transformation, which is uniquely defined owing to bijectivity of $\hat{\phi}$. The functional form of the approximant $\hat{\phi}$ reflects the modelling assumptions for the specific problem at hand. In [35], $\hat{\phi}$ is a piecewise quadratic approximation reflecting the model of a piecewise constant acceleration, in [41] a cubic spline model is used to represent a smooth phase variation, which proved to be successful for wind turbines signal analysis. The cubic spline model needs a priori knowledge of all the signal values, whereas the piecewise approximation can be updated as soon as new pulses become available from the encoder. For a machine operating at constant speed with small random, stationary fluctuation, Eq. (3) is written as:

$$
\phi: t \rightarrow \theta(t)=f(t)+r(t)=\Omega t+\int_{-\infty}^{t} v(u) d u
$$

with $\Omega$ constant operational speed. It is important to choose a model which represents the available knowledge of the problem and it is, at the same time, the simplest possible in order to prevent overfitting. In the case of Eq. (4), a piecewise linear approximation to $\phi$ might be appropriate and, due to less complexity of the linear model, more robust to estimation errors, albeit introducing discontinuities in the angular speed from one revolution of the shaft to another. With $r$ representing the ratio between trigger cycle and the fundamental CS1 cycle of interest, the linear piecewise approximation $\hat{\phi}_{L}$ to $\phi$ is obtained given the set of the $M$ encoder pulse arrival times $\mathbb{\tau}:\left(T_{p_{1}}, T_{p_{2}}, T_{p_{M}}\right)$ and the encoder angular spacing (or trigger cycle) $\Theta_{\text {trig }}=r \Theta$ as:

$$
\hat{\phi}_{L}: t \rightarrow \hat{\theta}(t)=\frac{\Theta_{\text {trig }}}{T_{p_{i+1}}-T_{p_{i}}} t+\hat{\theta}\left(T_{p_{i}}\right), \quad T_{p_{i}} \leqslant t<T_{p_{i+1}}, \quad i=(1,2, \ldots M-1)
$$

that is a set of piecewise linear transformations such that $\hat{\theta}(t)=1 / \lambda_{i} t+b_{i}$, with $1 / \lambda_{i}$ slope coefficient depending on the estimated (constant) speed during the $i$-th encoder interval and $b_{i}$ an offset corresponding to the initial phase of the $i$-th encoder interval, with $b_{1}$ conventionally set to 0 . Once the transformation $\phi$ has been estimated, the following step consists of interpolating the time-domain vibration signal to obtain its values for a constant angular increments grid. This is accomplished by inverting the mapping so to obtain $x(\hat{\theta})=y\left(\hat{\phi}^{-1}(\hat{\theta} ; \mathbb{T})\right)$. In doing that, care must be taken as the interpolation acts practically as a low-pass filter in the base-band. An extensive discussion of the effects of interpolation on the vibration signal can be found in [42].

\subsection{Synchronous averaging}

The basic idea of synchronous averaging is illustrated in Fig. 1, which shows the waveform $x_{S A}(\theta)$ obtained by synchronously averaging $N$ waveforms $x(\theta)$, indexed on the $y$-axis as $i=1,2 . . i . ., N$ and periodic on $2 \pi$ radians (where a full cycle corresponds, generally, to a shaft revolution) and corrupted by noise. As shown in Fig. 1, all the components of the signal which are not synchronous with the fundamental period get attenuated in the process, and it is indeed the case for the random noise components. In the limit of an infinite number of averages, the purely synchronous, periodic part of the signal is extracted (see Eq. (2)). As previously introduced in Section 1, under the assumption of cycloergodicity, SA is indeed a prac- 


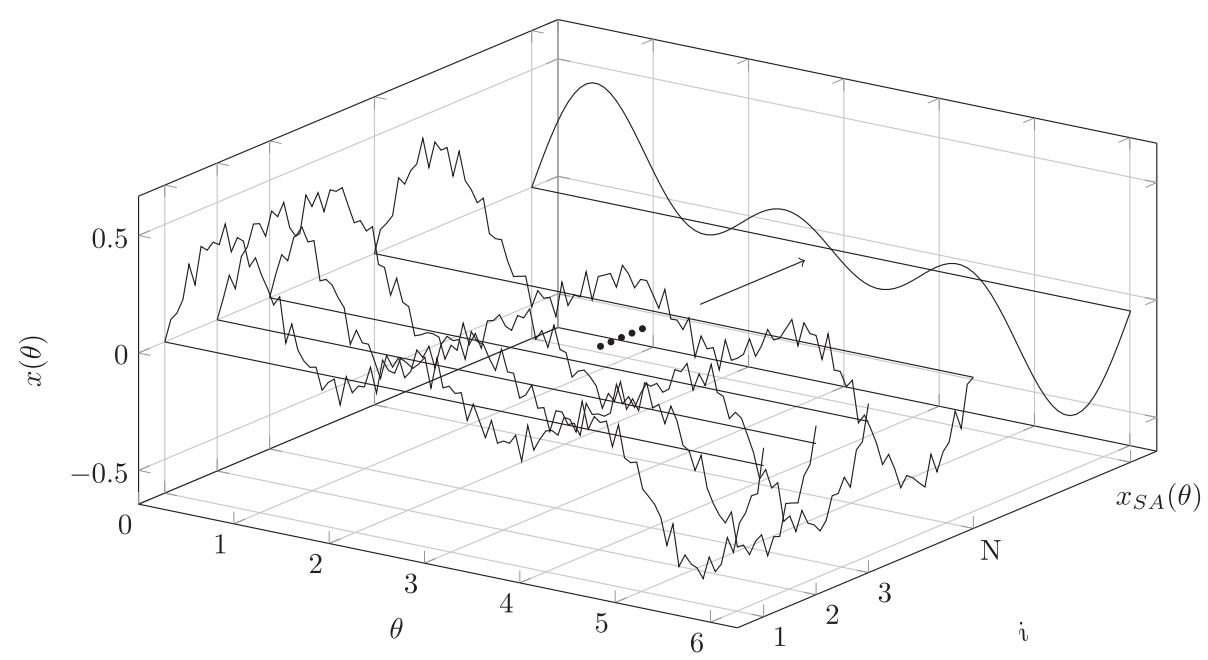

Fig. 1. Illustration of synchronous averaging procedure for the extraction of a periodic waveform. The noisy signal $x(\theta)$, which has components periodic in $\theta \in[0,2 \pi)$ is averaged $N$ times to produce the smooth synchronous waveform $x_{S A}(\theta)$.

tical estimation of the periodic mean of a CS signal, which is its first order cyclostationary part of cycle equal to the fundamental period used for averaging. The equation for SA of a signal $x(\theta)$ of fundamental cycle $\Theta$ reads in angle domain:

$$
S A[x(\theta)]_{\Theta}=\frac{1}{N} \sum_{i=0}^{N-1} x(\theta+i \Theta)
$$

Synchronous averaging is thus equivalent to applying a comb filter to the signal [6], which extracts the multiples of the reference harmonic. The number of averages controls the bandwidth of the lobes, the amount of noise rejection and the position of the notches of the filter. In fact, by Fourier transforming to the order domain $\gamma_{\Theta}=\gamma / \Theta$ (i.e. the angular-counterpart of the frequency domain variable normalized by the fundamental cycle of the signal), the SA reads:

$$
\mathcal{F}_{\theta \rightarrow \gamma_{\Theta}}\left(S A[x(\theta)]_{\Theta}\right)=X\left(\gamma_{\Theta}\right) \sum_{i=0}^{N-1} e^{j 2 \pi i \gamma_{\Theta}}=X\left(\gamma_{\Theta}\right) H_{S A}\left(\gamma_{\Theta}\right)
$$

where the symbol $j$ indicates the imaginary unit and $X\left(\gamma_{\Theta}\right)$ is the order domain representation of $x(\theta)$. Here the quantity $H_{S A}\left(\gamma_{\Theta}\right)$ acts as a transfer function which filters the angle-domain signal. It is easy to check that such a transfer function has unitary gain when the order variable $\gamma_{\Theta}$ takes integer values, i.e. when the Fourier-transformed angular variable is a multiple of the fundamental averaging cycle. Developing the summation in Eq. (7) with respect to the argument of the exponential, one has:

$$
H_{S A}\left(\gamma_{\Theta}\right)=\frac{\sin \left(\pi \gamma_{\Theta} N\right)}{N \sin \left(\pi \gamma_{\Theta}\right)} e^{j \pi \gamma_{\Theta}(N-1)}
$$

The resulting comb filter attenuates the signal which is not synchronous with the reference order, applying a gain lower than unity in the asynchronous regions of the spectrum. It can be shown that the stationary noise is attenuated by a factor $1 / \sqrt{N}[6]$. Eq. (8) shows that the parameter $N$ controls the selectivity of the filter and the attenuation of the non-synchronous components. Moreover, the position of the notches depends on the number of averages as well. Hence, a strong periodic component of a cycle, which is not multiple of the basic cycle of interest present in the signal, can be completely cancelled from the response only if it is possible to select a number of averages such that a notch gets located in correspondence of the frequency of such component. Further details on the interpretation of synchronous averaging as a comb filter are given in [38].

\subsection{Encoder random jitter error}

The encoder pulse time of arrival can be estimated from the encoder analogical signal, as detailed in [33]. An error on the pulse arrival times estimation arises from instrument imperfections (e.g. geometrical errors), electrical perturbations, physical phenomena and measurement limitations. The effect of these errors on angular resampling can be estimated by introducing the set $\hat{\mathbb{T}}$ such that every element $\widehat{T}_{p_{i}}$ in $\hat{\mathbb{T}}$ is equal to the sum of the corresponding element $T_{p_{i}}$ in the set of the pulse arrival times $\mathbb{T}$ and a jitter error $\epsilon_{i}$. In this study, systematic errors arising from geometrical instrument imperfections are neglected, and the attention is focused on the effect of the random errors. Each $\epsilon_{i}$ is assumed to be a random, uncorrelated 
jitter error, with identical probability density function (p.d.f.) $p\left(\epsilon_{i}\right)$. The approximant $\hat{\phi}$ to $\phi$ is estimated from the set $\widehat{T}$, resulting in approximation errors of the angle-time relationship which ultimately lead to distortion in the reconstructed vibration signal. This aspect of the problem is introduced in [24], where experimentation with decreasing precision in the keyphasor signal time of arrivals leads to progressively more deteriorated estimates of the order content of the resampled signal.

Fig. 2, similarly to [24], shows qualitatively a keyphasor signal sampled with a sampling frequency $f_{K P}$. The rate of sampling $f_{K P}$ at which the keyphasor is sampled determines the resolution of the keyphasor pulse arrival times. A pulse will be detected when the rising edge of the signal surpasses a threshold (dashed line in Fig. 2). Due to the resolution being limited from the keyphasor sampling frequency, there is indeed an uncertainty on the real pulse arrival time, which can be expressed as a random error. This is assumed to be the dominant contribution to the error in several industrial settings involving keyphasor signals consisting of one pulse per revolution of the trigger shaft. In this paper, an analytical formulation is provided and the effect of the random pulse arrival time errors $\epsilon_{i}$ on the estimated amplitude of the synchronous components is quantified. Monitoring systems require the design of acquisition configurations considering the duration of each acquisition and the sampling frequency for each channel. By predicting the effects of the jittering error on the estimates of the synchronous components as a function of the number of averages, the uncertainty extent, the considered averaging cycle length and the noise extent, it is possible to determine the acquisition duration and keyphasor sampling frequency which are necessary for an accurate estimation of the CS1 part of the signals of interests, providing information about both the expected attenuation for a given component and the variance of the estimates. Also, the outcome of the analysis could be employed for future research on the refinement of algorithms dealing with the estimation of the synchronous average in variable speed conditions, as the generalized synchronous average proposed in [43].

\section{Modelling the synchronous average under random jitter of the keyphasor}

\subsection{Previous work}

The effect of keyphasor signal timing accuracy on the computed order tracking procedure was first reported in [24], where it was experimentally shown that high sampling rate of the encoder signal produces cleaner order tracked signals. However, the paper was mainly concerned with the computed order tracking method, and the synchronous averaging procedure was not discussed. High sampling rates of the encoder signal were recommended, but no quantitative analysis is performed. In a different domain, statistics for time-domain cycle-average processes in presence of cycle-misalignment errors were studied in [44], concerned with ECG signals. A first attempt to model the effect of uncertainty in the pulse arrival times on synchronous average in the context of vibration monitoring was given in [25], where the effects of phase estimation errors in the proposed automatic tacho-less synchronous averaging procedure (derived extending the work published in [26]) were discussed. Specifically, the presented model for the $i$-th rotation period of a signal $x(t)$ considering a random estimation error $\epsilon_{i}$ of the tacho pulse instant at rotation $i$ was:

$$
x_{i}(t)=s\left(t-\epsilon_{i}\right)+n(t)
$$

where $s(t)$ is the deterministic part of the signal periodic with the period of interest and $n(t)$ the random and nonsynchronous sources. After an infinite number of averages, i.e. in the case of ideal synchronous averaging of Eq. (2), the extracted signal reads accordingly:

$$
m_{x}(t)=E\left[x_{i}(t)\right]=E\left[s\left(t-\epsilon_{i}\right)\right]
$$

and then, holding the commutativity of the Fourier transform applied to expectancy and considering that $m_{x}(t)$ is a periodic signal, its frequency representation reads:

$$
M_{x}(f)=E\left[S(f) e^{-j 2 \pi f \epsilon_{i}}\right]=S(f) E\left[e^{-j 2 \pi f \epsilon_{i}}\right]
$$

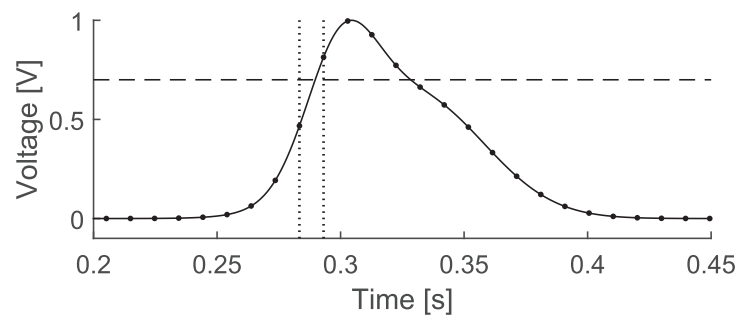

Fig. 2. Uncertainty on pulse arrival time due to keyphasor digital resolution. Continous line: analogical output; dots: discretized output; dashed horizontal line: trigger level; dotted vertical lines: uncertainty on pulse arrival time. 
Considering the $\epsilon_{i}$ independent, identically distributed (i.i.d.) with probability density function $p\left(\epsilon_{i}\right)$, Eq. (11) can be written as:

$$
M_{x}(f)=S(f) \Phi(f)
$$

With $\Phi(f)$ denoting the Fourier transform of $p\left(\epsilon_{i}\right)$, acting as a low-pass filter on the SA spectrum. The narrower is the probability density function of the random jitter error, the higher is the cut-off frequency of the low-pass filter (in the limit of zero jitter, the p.d.f. tends to a Dirac delta distribution, which has unit Fourier transform and produces no attenuation in all the frequency domain). Formally, the model is equivalent to summing cycles of the signal with a random phase delay $\epsilon_{i}$ and reproduces the same attenuation model obtained in [4] for the quasi-periodic bearing excitation. However, such modelling is inadequate for the computed order tracking and synchronous average procedures detailed in Section 2. The uncertainty in pulse arrival times determines in facts a reconstruction error in the angular resampling step, which results in distortion of the original waveform, rather than in constant random phase shifts between different revolutions.

\subsection{Proposed model}

According to the formulation presented in Section 2, the effect of keyphasor inaccuracy is modelled as random i.i.d. errors on the pulse arrival times. The vibration signal is resampled using computed order tracking based on the estimated timeangle relation:

$$
x(\hat{\theta})=x(\hat{\phi}(t ; \hat{\mathbb{T}}))
$$

As discussed in Section 2.1, a first approximation error depends on the fact that a set of model functions are used to reconstruct the time-angle relationship in those intervals for which no keyphasor is available. A second approximation error is instead introduced by using the set of estimated pulse arrival times $\mathbb{\pi}$ in place of the set of the actual pulse arrival times T. Consequently, when estimating the synchronous average from Eq. (6), the cyclic average of $x(\hat{\theta})$ is estimated in place of the cyclic average of $x(\theta)$. If the fundamental cycle is $\Theta$, the synchronous average of the angularly resampled signal reads, considering $N$ fundamental periods (typically shaft revolutions):

$$
S A[x(\hat{\theta})]_{\Theta}=\frac{1}{N} \sum_{i=0}^{N-1} x(\hat{\theta}+i \Theta)
$$

where the unique difference with respect to Eq. (6) lies in having approximated $\phi$ with $\hat{\phi}$, hence mapping the signal $y(t)$ to $x(\hat{\theta})$. When ideal pulse arrival times are used and the model for the time-angle relation is exact, for example when a quadratic model is used to approximate a constant acceleration signal, then the mapping is exact and $\hat{\theta}=\theta$. Similarly, the mapping is exact when the rotational speed is constant within the considered interval and a linear model is adopted for defining the angle-sampling grid. When the mapping is not exact, the resampling results in a distortion of the signal which depends on the difference between approximated and real angle progression with time. Since the real angle progression is unknown, such distortion cannot be recovered and accounted for. The following assumptions are made:

(i) the rotational speed is sufficiently stable and only subjected to small, random fluctuations during an acquisition, therefore allowing to represent the true angle progression in the form of Eq. (4);

(ii) the random speed fluctuations are small in amplitude w.r.t. the mean rotational speed and represented by a stationary, band-limited stochastic process during the acquisition, on account of high rotor inertia;

(iii) short-period speed fluctuations, such as those faster than the trigger period, are small in amplitude.

Assumptions (i), (ii), and (iii) are in general a good approximation for the behaviour of any rotor system running at constant operational speed, with high inertia. Based on assumptions (i) and (iii), it is possible to approximate $\phi$ with $\hat{\phi}_{L}$ from Eq. (5) with negligible error. This amounts to assuming that $\hat{\phi}_{L}(t ; \mathbb{T})=\hat{\theta} \approx \theta$. Under the declared conditions, the computed order tracking allows an almost perfect mapping and the synchronous average is written as in Eq. (6). The extracted synchronous components have therefore unit gain in this setting. Furthermore, small speed fluctuations limit the phase and amplitude modulation effects from the transfer path [45].

Knowing $\mathbb{T}$ a priori under (i), (ii) and (iii) would favour smoother approximants than the piecewise linear law discussed in Section 2.1. However, not only the piecewise linear approximation is sufficiently accurate under the stated hypothesis, but it can also be expected to be more robust when $\hat{\mathbb{T}}$ is used in place of $\mathbb{T}$, being the linear model simpler and less prone to error propagation owing to the smaller number of parameters to be estimated w.r.t. higher order interpolating functions. The conditions under which the chosen approximation performs better than more complex models for the angle progression reconstruction are not discussed in the paper, where the effect of pulse time uncertainty is emphasized with respect to the effect of the approximation errors due to the choice of the functional form for $\hat{\phi}$. Depending on the adopted angle progression model, the effect of the errors on the pulse arrival times on the reconstructed signal is different. Holding the assumptions 
(i), (ii), (iii) and adopting the linear piecewise model from Eq. (5), the errors translate into stretching and offset in the piecewise linear components related to each revolution, which can be accounted for as random variations of the estimated coefficients $\hat{\lambda}_{i}$ and $\hat{b}_{i}$ with respect to their actual values $\lambda_{i}$ and $b_{i}$. The $i$-th trigger cycle of the vibration signal resampled in the angle domain is then:

$$
x_{i}(\hat{\theta})=x\left(\frac{1}{\hat{\lambda}_{i}} t+\hat{b}_{i}\right)=y\left(\hat{\lambda}_{i}\left(\hat{\theta}-\hat{b}_{i}\right)\right), \quad \widehat{T}_{p_{i}} \leqslant t<\widehat{T}_{p_{i+1}}, \quad 0 \leqslant \hat{\theta}<\Theta_{\text {trig }}
$$

where the transformation $\hat{\phi}$ brings the values of $t$ in $\hat{\theta}$. It is known that the vibration signal of interest has components which are periodic (or CS1) in $\theta$, as detailed in the previous sections. Such periodicities are not preserved in $x(\hat{\theta})$ as the periodic components are not fully recovered due to the inexact mapping from the time domain. However, being the random errors unknown, the exact transformation cannot be estimated and the resampling step cannot be corrected. Consequently, the periodic components need to be estimated from $x(\hat{\theta})$. It is now worthwhile to make explicit the effect of the errors on the ideal pulse arrival times on the reconstructed signal. From Eqs. (5) and (15), replacing $T_{p_{i}}$ with $\widehat{T}_{p_{i}}=T_{p_{i}}+\epsilon_{i}$, after making the offset explicit:

$$
x_{i}(\hat{\theta})=y\left(\left(\lambda_{i}+\frac{\epsilon_{i+1}-\epsilon_{i}}{\Theta_{\text {trig }}}\right) \hat{\theta}+\sum_{q=1}^{i-1} \lambda_{q} \Theta_{\text {trig }}+\epsilon_{i}\right), \quad 0 \leqslant \hat{\theta}<\Theta_{\text {trig }}
$$

In the frame of condition monitoring applications, it is important to estimating the amplitude of specific periodic components, since it is associated to the energy of those dynamics synchronous with the cycle of interest, which provides diagnostic information on the machine.

In the following, under hypothesis (ii), it is shown that the mapping error results in an attenuation of the synchronous components amplitudes in the signal, with statistics connected to those of the jitter error. Taking $\theta$ as the angular variable connected to the shaft of interest, a periodic signal of order $\gamma^{*} \in \mathbb{N}$ in $\theta$ and initial phase $\eta_{0}$ is represented in analytical form as $x(\theta)=e^{j 2 \pi\left(\gamma^{*}+\eta_{0}\right)}$, then each fundamental cycle is obtained as:

$$
x_{k}(\theta)=e^{j 2 \pi\left(\gamma^{*} \theta+\eta_{0}+(k-1) \Theta\right)}, \quad 0 \leqslant \theta<\Theta
$$

Considering Eqs. (16) to (17), introducing non-ideal pulse arrival times it results:

$$
x_{k_{i}}(\hat{\theta})=e^{j 2 \pi\left(\gamma^{*}\left(1+\frac{\epsilon_{i+1}-\epsilon_{i}}{\Theta_{\text {trig }} i_{i}}\right)\left(\hat{\theta}+\left(k_{i}-1\right) \Theta\right)+\eta_{0}+\frac{\epsilon_{i}}{\hat{i}_{i}} \gamma^{*}\right)}, \quad 0 \leqslant \hat{\theta}<\Theta
$$

being $r \in \mathbb{N}$ and $k_{i}$ the index of the fundamental cycle within the $i$-th trigger cycle. The parameter $\gamma^{*}$ allows one to select any integer multiple of the cycle of interest. In the most common case in which $r$ is not an integer, there are fundamental cycles which fall in-between different trigger cycles. For these cycles, Eq. (18) would split in two subdomains such that the $i$ dependent parameters would take a different value in each of the subdomains. However, this complication can be simplified in the averaging process by assigning these cycles to the trigger cycle in which the majority of the fundamental cycle domain falls. It is shown in Section 4.1 that this approximation allows computing practical analytical solutions when $r$ is large enough w.r.t. the unity and a sufficient number of averages is performed. Taking the Fourier transform of Eq. (18) to order domain, the following is obtained:

$$
X_{k_{i}}(\gamma)=D_{\Theta}\left(\gamma-\gamma^{*}-\gamma_{i}\right) e^{j 2 \pi\left(\eta_{0}+\left(k_{i}-1\right) \Theta\left(\gamma^{*}+\gamma_{i}\right)+\frac{\epsilon_{i}}{\lambda_{i}} \gamma^{*}\right)}
$$

in which the sifting property of the Dirac's delta distribution was used and:

$$
\begin{aligned}
& \gamma_{i}=\frac{\gamma^{*}}{\Theta_{\text {trig }} \lambda_{i}}\left(\epsilon_{i+1}-\epsilon_{i}\right) \\
& D_{\Theta}(x)=\Theta \frac{\sin (\pi x \Theta)}{\pi x \Theta} e^{-j \pi x \Theta}
\end{aligned}
$$

being $D_{\Theta}(\cdot)$ the order domain transform of the rectangular window of width $\Theta$ centered in $\Theta / 2$. Eq. (19) shows that the effect of random error on the pulse times is that of a phase and order shift which is different per each cycle depending on the statistics of the random error through the $\epsilon_{i}$ and on the statistics of the speed fluctuation through the $\lambda_{i}$. Finally, the synchronous average operation aimed to extract the fundamental cycle can be written as:

$$
x_{S A}(\hat{\theta}) \approx \frac{1}{N_{t}} \sum_{i=1}^{N_{t}}\left(\frac{1}{N_{i}} \sum_{k=1}^{N_{i}} e^{j 2 \pi\left(\Theta\left(\gamma^{*}+\gamma_{i}\right)(k-1)\right)}\right) e^{j 2 \pi\left(\left(\gamma^{*}+\gamma_{i}\right) \hat{\theta}+\frac{\epsilon_{i}}{\lambda_{i}} \gamma^{*}+(i-1) N_{i} \Theta \gamma^{*}\right)}, \quad 0 \leqslant \hat{\theta}<\Theta
$$

in which $N_{i}$ is the number of fundamental cycles assigned to trigger cycle $i, N_{t}$ is the number of averaged trigger cycles, and $\eta_{0}$ was set to zero for convenience (the result for an arbitrary initial phase factor can be recovered simply multiplying by $e^{j \eta_{0}}$ ). 
Setting the jitter error to zero in Eq. (22) and considering $r \in \mathbb{N}$, that is the case in which $N_{i}=N$ and each trigger cycle contains an integer number of fundamental cycles, it is possible to determinate indeed that the result equals the synchronous average of $N_{t} N$ fundamental cycles, recovering the results from Section 2.2. Eq. (22) exactly holds if $r \in \mathbb{N}$ and approximatively if $r \in \mathbb{R}$, with $r \geqslant 1$. When $0<r<1$, the fundamental cycle is slower than the trigger cycle, therefore discontinuities exist within each averaged cycle and Eq. (18) is inadequate to modelling the synchronous average.

From a practical point of view, averaging on longer cycles than the trigger cycle means that the reconstructed signal cycles would involve one or more discontinuities in the angle progression law within each cycle. Hence, if averaging is required for extracting low frequency CS1 components and a piecewise angular resampling is adopted under hypotheses (i), (ii) and (iii), it is advised to downsample the pulse arrival times so to produce smoother estimates of the angle progression within each cycle of interest. Transforming Eq. (22) to the order domain and considering the finite domain spanning one fundamental cycle, it is possible to write:

$$
\left.X_{S A}(\gamma) \approx \frac{1}{N_{t}} \sum_{i=1}^{N_{t}}\left(\frac{1}{N_{i}} \sum_{k=1}^{N_{i}} e^{j 2 \pi\left(\Theta\left(\gamma^{*}+\gamma_{i}\right)(k-1)\right)}\right) e^{j 2 \pi\left(\frac{\epsilon_{i}}{\lambda_{i}} \gamma^{*}+(i-1) N_{i} \Theta \gamma^{*}\right.}\right) D_{\Theta}\left(\gamma-\gamma_{i}-\gamma^{*}\right)
$$

Again, when the jitter is null, Eq. (23) boils down to the product of the two terms:

$$
X_{S A}(\gamma) \approx \frac{1}{N_{t}} \sum_{i=1}^{N_{t}}\left(\frac{1}{N_{i}} \sum_{k=1}^{N_{i}} e^{j 2 \pi \Theta \gamma^{*}(k-1)}\right) e^{j 2 \pi\left((i-1) N_{i} \Theta \gamma^{*}\right)} D_{\Theta}\left(\gamma-\gamma^{*}\right)
$$

which can be recognized as the product of a periodic component of frequency $\gamma^{*}$ observed on the cycle duration $\Theta$ and an attenuation factor depending on the number of averaged revolutions, the ratio of the fundamental cycle duration to the trigger duration (through the coefficients $N_{i}$ ) and the product of the order of interest and the fundamental cycle duration. Through Eq. (24), it is recognized that the transformation acts as a filter with unit gain for components $\gamma^{*}$ which are synchronous with respect to the fundamental cycle $\Theta$, recovering again the results from Section 2.2. It is also interesting to highlight that the synchronous components extracted in the case of Eq. (24) do not interact with each other, owing to the periodicity of the rectangular window transform. Eq. (23) shows that the random phase and order shifts for each cycle result in an attenuation factor even for the synchronous components. In fact, the condition on the product between the fundamental cycle and the order to be integer is no longer sufficient to enforce unit gain due to the additional, random terms. Furthermore, for $\gamma=\gamma^{*}$, the residual order shift $\gamma_{i}$ results in the periodic component term per each cycle being attenuated according to the scalloping loss of the rectangular window transform. For multiples of $\Theta$ such that $\gamma \neq \gamma^{*}$, there is residual noise resulting in the extracted orders influencing each other in the synchronous average. This is particularly relevant when there are strong gearmesh orders in rotating machinery, since the leakage might mask the amplitude of the surrounding modulation sidebands. It must be observed that the expression in Eq. (23) is a continuous function of $\gamma$, wehereas the synchronous average on a digital computer results in a discrete periodic function, which is obtained by sampling Eq. (23). Such a sampling can be made arbitrary from a discrete sequence by zero-padding.

\subsection{Approximation to the distribution of the amplitude attenuation}

Eq. (23) can be used to study the distribution of the amplitude attenuation for a given CS1 component in the signal by varying the number of averages, the ratio of the trigger cycle duration to the fundamental cycle duration, the probability distribution for the random error and the autocorrelation function for the speed fluctuation. The possibility of quickly evaluating Eq. (23) can be used to perform extensive Monte Carlo simulations under different settings, obtaining the distribution of the amplitude attenuation of the estimated synchronous components under hypotheses (i), (ii), and (iii) from Section 3.2 for any combination of the input parameters, without having to actually work with discrete signals and going through the computed order tracking resampling steps at each iteration. Monte Carlo simulations are however expensive to evaluate for all the combination of parameters. Hence an analytical approximation to the distribution of the extracted synchronous amplitudes is obtained for the synchronous average process exploiting central limit theorems, in order to obtain more complete information over the entire domain of interest. The following quantities are defined:

$$
\begin{aligned}
& \bar{\epsilon}_{i}=\frac{\epsilon_{i}}{\lambda_{i}} ; \\
& \overline{\bar{\epsilon}}_{i}=\frac{\epsilon_{i+1}}{\lambda_{i}}
\end{aligned}
$$

and assuming independency of the keyphasor errors $\epsilon_{i}$, it holds that $\bar{\epsilon}_{i}$ is independent of $\overline{\bar{\epsilon}}_{i}$, whereas $\overline{\bar{\epsilon}}_{i}$ is correlated to $\bar{\epsilon}_{i+1}$ through the autocorrelation function of the speed variations. By specifying the correlation model, the distribution for $\lambda_{i}$ and the keyphasor error model, the random error on the estimated synchronous components in the spectrum of the synchronous averaged signal can be simulated in Eq. (23). An approximate analytical solution to the amplitude distribution can be obtained under the following conditions:

(iv) the speed variations are negligible compared to the error, i.e. $\lambda_{i} \approx \lambda$; 
(v) the $\epsilon_{i}$ variables are uniformly, identically distributed and independent as $U_{[-\Delta / 2, \Delta / 2]}$, that is a uniform distribution, with center zero and $\operatorname{span} \Delta$.

These choices are not mandatory for the numerical simulations through the model developed in Section 3.2, however they guarantee: (a) that the effect of the keyphasors uncertainty can be studied independently from the speed fluctuation statistics and (b) that the uncertainty is limited within an interval, hence preventing an unphysical permutations of the keyphasor pulses. Furthermore, they allow deriving a practical analytical approximation to the distribution of the extracted amplitude as a series expansion. As a remark, taking a constant speed signal, angle and time domain would be linearly related by a constant stretching factor for the entire signal, and a linear transformation would suffice to describe the time-angle relationship. Besides, the angular resampling is not necessary in that case, since the linear phase would preserve CS1 components in the time domain. Hence, the approximated distribution has the only function of shedding light on the role of keyphasor signal accuracy in absence of other sources of uncertainty. However, it will be shown in Section 5 that the distributions obtained under (iv) reasonably approximate the operational scenarios under (v). From Eq. (23), developing the exponent sum and parametrizing the order such that $\Theta=1$ is the cycle of interest, with $\gamma^{*} \in \mathbb{N}$ indicating that integer (synchronous with the averaging cycle) orders are considered:

$$
X_{S A}\left(\gamma^{*}\right)=\frac{(-1)^{\gamma^{*}} e^{-j \pi \gamma^{*}}}{N_{t}} \sum_{i=0}^{N t-1}(-1)^{N_{i}\left(1+\gamma^{*}\right)} \operatorname{sinc}\left(N_{i} \pi \gamma_{i}\right) e^{j 2 \pi\left(\bar{\epsilon}_{i} \gamma^{*}+\frac{N_{i} \gamma_{i}}{2}\right)}
$$

By making use of Euler's identity, from Eq. (26), the absolute value of the synchronous averaged unit amplitude component $\gamma^{*}$ reads:

$$
\left|X_{S A}\left(\gamma^{*}\right)\right|=\sqrt{\left(\frac{1}{N_{t}} \sum_{i}(-1)^{N_{i}\left(1+\gamma^{*}\right)} \cos \left(k_{i}\right) \operatorname{sinc}\left(\tau_{i}\right)\right)^{2}+\left(\frac{1}{N_{t}} \sum_{i}(-1)^{N_{i}\left(1+\gamma^{*}\right)} \sin \left(k_{i}\right) \operatorname{sinc}\left(\tau_{i}\right)\right)^{2}}
$$

With:

$$
\begin{aligned}
& k_{i}=2 \pi\left(\bar{\epsilon}_{i} \gamma^{*}+\frac{N_{i} \gamma_{i}}{2}\right) \\
& \tau_{i}=N_{i} \pi \gamma_{i}
\end{aligned}
$$

Using (iv), (v), the random variables $\bar{\epsilon}_{i}, \overline{\bar{\epsilon}}_{i}$ can be rewritten so that $\epsilon_{i} / \lambda=\tilde{\epsilon}_{i}, \epsilon_{i+1} / \lambda=\tilde{\epsilon}_{i+1}$, and owing to the linearity of the transformation, they turn to be i.i.d. uniform random variables distributed as $U_{[-\Delta / 2 \lambda, \Delta / 2 \lambda]}$. The stretching factor $\lambda$ characterizes the time to angle transformation and relates the cycles in time domain to the cycles mapped in the angle domain. In order to solve the statistics, it is convenient to parametrize the error with respect to the fundamental cycle in time domain, getting rid of the stretching factor. The $i$-th functions of the random variables $\tilde{\epsilon}_{i}, \tilde{\epsilon}_{i+1}$ which appear respectively inside the first and the second squared summations under the square root in Eq. (27) are denoted $g_{1 i}$ and $g_{2 i}$, and the index $q=1,2$ is introduced. The dependency of $g_{q i}$ on the random variables is omitted from the text for easiness of notation. To compute the statistics of the amplitude, that is the distribution of $\left|X_{S A}\left(\gamma^{*}\right)\right|$, central limit theorems can be exploited so as to approximate the real and the imaginary parts with normal distributions. Then, the sum of squares falls in the family of quadratic forms in normal variables, for which an approximated distribution can be derived based on the known statistical cumulants and moments.

The two squared terms under the root sign in Eq. (27) are both in the form of the average of a series of random variables. The random variables $g_{q i}$ have finite variance and fulfil mixing conditions such that the central limit theorem under strongmixing condition holds for $N_{t}$ large enough and $r \in \mathbb{N}$. In fact, $g_{q i}, g_{q j}$ are mutually independent for every $|i-j|>1$, and the set of values taken from $N_{i}$ is finite, i.e. $N_{i} \in A$, a subset of $\mathbb{N}$ with finite cardinality depending on the value of $r$ and on the number of trigger cycles. The cardinality of $A$ is always one when $r \in \mathbb{N}$, hence the variables in the sums are identically distributed. For the general case of $r \in \mathbb{R}^{+}$, the sums over $i$ of the $g_{q i}$ are composed of terms which are not identically distributed. By the Cramír-Wold theorem, it holds for $\mathbf{g}$ :

$$
\frac{1}{\sqrt{N_{t}}}(\mathbf{g}-E[\mathbf{g}]) \rightarrow \mathcal{N}_{2}(\mathbf{0}, \mathbf{\Sigma})
$$

Where:

$$
\mathbf{g}=\left[\sum_{i} g_{1 i}, \sum_{i} g_{2 i}\right]^{\top}=\left[g_{1}, g_{2}\right]^{\top}
$$

The symbol $\mathcal{N}_{2}$ denotes the multivariate normal distribution with two degrees of freedom and $\boldsymbol{\Sigma}$ the associated covariance matrix. The elements of $\boldsymbol{\Sigma}$ are given as: 


$$
\begin{aligned}
& \Sigma_{q q}=\frac{1}{N_{t}} \operatorname{Cov}\left(g_{q}, g_{q}\right)=\frac{1}{N_{t}}\left(\sum_{i=1}^{N_{t}} \operatorname{Var}\left(g_{q i}\right)+2 \sum_{1 \leqslant i<j<N_{t}} \operatorname{Cov}\left(g_{q i}, g_{q j}\right)\right) \\
& \Sigma_{12}=\Sigma_{21}=\frac{1}{N_{t}} \operatorname{Cov}\left(g_{1}, g_{2}\right)
\end{aligned}
$$

Specifically, the Central Limit Theorem (CLT) holds for the strong mixing sequences representing the components of $\mathbf{g}$ [46], and subsequently the Cramír-Wold device can be used to establish that linear combinations behave likewise. Considering the distribution of the absolute value, it holds:

$$
\left|X_{S A}\left(\gamma^{*}\right)\right|^{2}=\mathbf{g}^{\top} \mathbf{I g}
$$

The random vector $\mathbf{g}$ is approximately distributed as a bivariate normal $\mathcal{N}_{2}(E[\mathbf{g}], \mathbf{\Sigma})$ according to Eq. (30), and the quantity in Eq. (34) is a quadratic form of normal variables. Such distributions are widely studied in literature [47]. As for this work, the probability density function was approximated using the moment-based method proposed in [47]. The procedure is outlined in Section $A$ and requires the computation of the expected values vector $E[\mathbf{g}]$ and of the covariance matrix $\Sigma$, which are easily obtained after computing the expected value and covariance terms for the functions $g_{q i}$. Let $x=\epsilon_{i} \gamma^{*}$ and $y=\epsilon_{i+1} \gamma^{*}$, and let (iv), (v) hold with the errors parametrized with respect to the fundamental cycle. By $(\mathrm{v}), p_{X, Y}(x, y)=p_{X}(x) p_{Y}(y)$ where $p_{\chi}(\cdot)$ denotes the probability density function of the random variable $\chi$. The expected values read then:

$$
E\left[g_{q i}\right]=\frac{1}{\Delta^{2}} \int_{-\frac{\Delta}{2}}^{\frac{\Delta}{2}} \int_{-\frac{\Delta}{2}}^{\frac{\Delta}{2}} g_{q i}(x, y) d x d y, \quad q=1,2
$$

Under the mentioned conditions, the integrals from Eq. (35) are written as:

$$
\begin{aligned}
& E\left[g_{1 i}\right]=\frac{-1^{N_{i}\left(1+\gamma^{*}\right)}}{\Delta^{2}} \int_{-\frac{\Delta}{2}}^{\frac{\Delta}{2}} \int_{-\frac{\Delta}{2}}^{\frac{\Delta}{2}} \cos \left(\pi\left(2 x+\frac{N_{i}}{r}\right)(y-x)\right) \operatorname{sinc}\left(\frac{\pi N_{i}}{r}(y-x)\right) d x d y \\
& E\left[g_{2 i}\right]=\frac{-1^{N_{i}\left(1+\gamma^{*}\right)}}{\Delta^{2}} \int_{-\frac{\Delta}{2}}^{\frac{\Delta}{2}} \int_{-\frac{\Delta}{2}}^{\frac{\Delta}{2}} \sin \left(\pi\left(2 x+\frac{N_{i}}{r}\right)(y-x)\right) \operatorname{sinc}\left(\frac{\pi N_{i}}{r}(y-x)\right) d x d y
\end{aligned}
$$

The elements of the expected value vector can hence be computed from Eq. (30), after the values from Eqs. (36) and (37) are obtained. The elements of the covariance matrix in Eqs. (32) and (33) are obtained similarly by integrating the squared values and considering the weak dependency terms. In the following, the cases of integer ratio is considered, and expressions for the required terms are developed. Albeit the formulation allows for obtaining the expressions in case of non-integer ratio those are not necessary for the results reported in this paper. A closed form solution for the expectations could be obtained for $N_{i} \in \mathbb{N}$, which implies also $N_{i} / r=1$. In this specific case, Eqs. (36) and (37) have the following solutions:

$$
\begin{aligned}
& E\left[g_{1 i}\right]=\frac{1}{(\pi \Delta)^{2}}\left(-\gamma_{E} \cos (\pi \Delta)+\cos (\pi \Delta) \operatorname{Ci}(2 \pi \Delta)-\cos (\pi \Delta) \log (2 \pi \Delta)+\sin (\pi \Delta) \operatorname{Si}(2 \pi \Delta)\right) \\
& E\left[g_{2 i}\right]=0
\end{aligned}
$$

where $\mathrm{Ci}(\cdot)$ and $\operatorname{Si}(\cdot)$ are the cosine and sine integral functions, and $\gamma_{E}$ is the Euler's gamma constant. Considering that the random variables take small values bounded in the region $[-\Delta / 2 ; \Delta / 2] \times[-\Delta / 2 ; \Delta / 2]$, the integrals in Eqs. (36) and (37) can be approximately expressed by expanding the sine cardinal function in power series and truncating the expansion to the second-order. Using the trigonometric identities for sums of angles, considering the extremes of integration and observing that $\cos (\cdot)$ is a bounded function, it is possible to show that the residual $q(x, y)$ integrates to a small error in the considered region. Therefore, the following approximation for the expected value is given:

$$
E\left[g_{1 i}\right] \approx \frac{8-(\pi \Delta)^{2}-8 \cos (\pi \Delta)}{3(\pi \Delta)^{2}}
$$

Eq. (40) turns to be more practical when studying the approximate analytical solutions for the moments of the quadratic form in Eq. (34). Similarly, approximate expressions for the covariance terms are given as:

$$
\begin{aligned}
& \sigma_{11 i i}^{2} \approx \frac{4 \pi^{6} \Delta^{6}-90 \pi^{4} \Delta^{4}+1710 \pi^{2} \Delta^{2}-1305 \cos (2 \pi \Delta)+1305}{4320 \pi^{2} \Delta^{2}}-\frac{\left(\pi^{2} \Delta^{2}+8 \cos (\pi \Delta)-8\right)^{2}}{9 \pi^{4} \Delta^{4}} \\
& \sigma_{22 i i}^{2} \approx \frac{4 \pi^{6} \Delta^{6}-150 \pi^{4} \Delta^{4}+2610 \pi^{2} \Delta^{2}+1305 \cos (2 \pi \Delta)-1305}{4320 \pi^{2} \Delta^{2}} \\
& \sigma_{12 i i}^{2} \approx 0
\end{aligned}
$$


Further, the non-zero dependency terms are identical for every $|i-j|=1$ and are given as:

$$
\begin{aligned}
\sigma_{11 i j}^{2} \approx & -\frac{32}{3 \pi^{4} \Delta^{4}}+\frac{128 \cos (\pi \Delta)}{9 \pi^{4} \Delta^{4}}-\frac{32 \cos (2 \pi \Delta)}{9 \pi^{4} \Delta^{4}}+\frac{113 \sin (\pi \Delta)}{72 \pi^{3} \Delta^{3}}-\frac{113 \sin (2 \pi \Delta)}{144 \pi^{3} \Delta^{3}}+\frac{\pi^{2} \Delta^{2}}{180}+\frac{241}{72 \pi^{2} \Delta^{2}}+ \\
& -\frac{\pi^{2} \Delta^{2} \cos (\pi \Delta)}{1080}-\frac{241 \cos (\pi \Delta)}{72 \pi^{2} \Delta^{2}}+\frac{1}{108} \pi \Delta \sin (\pi \Delta)-\frac{47 \sin (\pi \Delta)}{72 \pi \Delta}+\frac{1}{6} \cos (\pi \Delta)-\frac{7}{36} \\
\sigma_{22 i j}^{2} \approx & \frac{3\left(4 \pi^{5} \Delta^{5}-100 \pi^{3} \Delta^{3}+1430 \pi \Delta+565 \sin (2 \pi \Delta)\right)+10\left(2 \pi^{4} \Delta^{4}-51 \pi^{2} \Delta^{2}-339\right) \sin (\pi \Delta)}{2160 \pi^{3} \Delta^{3}} \\
& +\frac{-2 \pi \Delta\left(\pi^{4} \Delta^{4}-180 \pi^{2} \Delta^{2}+2145\right) \cos (\pi \Delta)}{2160 \pi^{3} \Delta^{3}} \\
\sigma_{12 i j}^{2} \approx & 0
\end{aligned}
$$

where $\sigma_{l m i j}^{2}$ indicates the covariance between $g_{l i}$ and $g_{m j}$. Closed form expressions for the solutions of the integrals corresponding to the diagonal elements of the covariance matrix were not derived. Fig. 3 shows the non-zero expected value (left) and the covariance matrix (right) components as a function of the uncertainty extent $\Delta$ according to the obtained expressions. In the adopted parametrization, the error $\Delta$ is normalized with respect to the fundamental cycle, and hence values higher than 0.5 would correspond to an uncertainty greater than the semi-duration of a cycle. Increasing the uncertainty extent leads to increased variance of both the functions of random variables $g_{q i}$. The dominant contribution to the variance in the sum of Eq. (27) comes from the $g_{2 i}$, whereas the dominant contribution to the mean value is related to the $g_{1 i}$. From Fig. 3, it is seen that the approximation for the expected value of $g_{1 i}$ results in an error growing with the uncertainty extent, as expected from the truncation to the second order of the function expansion around small uncertainty extent values. Nevertheless, the relative error keeps very small in the application domain, clearing the use of the more practical formula from Eq. (40) for the expected value without significant loss in accuracy.

\subsubsection{Effect of the noise}

From Eqs. (27) and (51), it is possible to derive the effect of additive, uncorrelated Gaussian noise on the estimates of the attenuation. Fourier transforming a centered Gaussian noise yields a circularly distributed complex noise, with the real and imaginary part of which equally accounting for the variance. By the properties of the normal distribution, the real part and the imaginary part in Eq. (27) under additive Gaussian noise of variance $\sigma_{n}^{2}$ result to be still normally distributed with the same mean and an additional term $\sigma_{n}^{2} / N$ on the diagonal of the covariance matrix. Considering the diagonal form obtained for the covariance matrix and the results for the expected value vector, the first two cumulants of the quadratic form read, from Eq. (51):

$$
k(1)=\Sigma_{11}+\Sigma_{22}+E\left[g_{1 i}\right]^{2}
$$

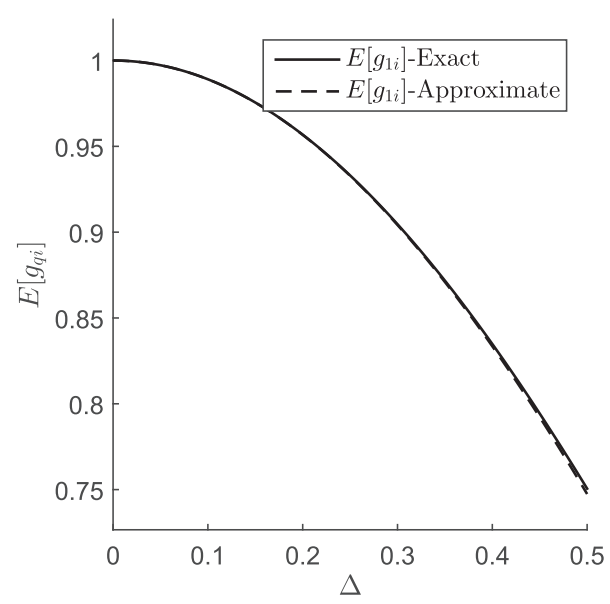

(a)

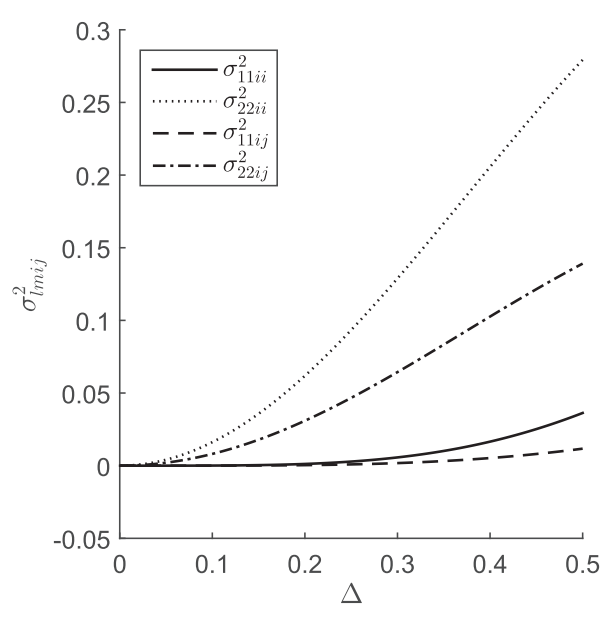

(b)

Fig. 3. Expected value and covariance matrix values for the $g_{q i}$ functions varying $\Delta-$ (a) Expected value for $g_{1 i}$, exact solution and second order approximation; (b) Components of the covariance matrix $\sigma_{l m i j}^{2}$ for $g_{q i}$ functions and dependency terms for $|i-j|=1$. 


$$
k(2)=4\left(\frac{\left(\Sigma_{11}+\Sigma_{22}\right)^{2}}{2}+E\left[g_{1 i}\right]^{2} \Sigma_{11}\right)
$$

Consequently, the cumulants under additive Gaussian noise read:

$$
\begin{aligned}
& k_{n}(1)=\Sigma_{11}+\Sigma_{22}+\frac{\sigma_{n}^{2}}{N^{2}}+E\left[g_{1 i}\right]^{2} \\
& k_{n}(2)=2\left[\left(\Sigma_{11}+\Sigma_{22}+\frac{\sigma_{n}^{2}}{N^{2}}\right)^{2}+2 E\left[g_{1 i}\right]^{2}\left(\Sigma_{11}+\frac{\sigma_{n}^{2}}{2 N^{2}}\right)\right]
\end{aligned}
$$

A deep discussion on the effect on higher cumulants is out of the scope of this paper. According to Eqs. (49) and (50), the additive noise has an impact on the estimated expected value of the squared amplitude of the synchronous harmonic of interest, and on its variance. In high signal to noise ratio conditions (i.e. $\sigma_{n}^{2} \ll 1$, since the amplitude of the harmonic component was normalized to unity), the latter is indeed more relevant, being the third term in Eq. (49) small with respect to the fourth term. Hence, the main effect of the additive noise consists of an increased dispersion in the distribution of the estimated amplitude.

\section{Numerical analysis}

\subsection{Numerical validation of the model}

In this section, it is shown that the model introduced in Section 3.2 allows to reproduce the results of the computed order tracking and synchronous average as described in Section 2. The model is validated as a surrogate for the digital angle synchronous average procedure and can then be used to infer the statistical properties of the synchronous signal as it is extracted. As mentioned, hypothesis (iv) and (v) from Section 3.3 are used in the numerical examples, in order to focus on the effect of the pulse time uncertainty independently from the statistics of the speed fluctuations. The case of a single harmonic signal is first studied, then an extension to a general multi-harmonic case considering two harmonics is presented. One step of the Monte Carlo iterations consists of generating a digital signal in time domain, simulating a vibration acquisition, then order-tracking it using a simulated reference keyphasor signal from which the exact pulse times are extracted, and then obtaining the synchronous average of the cycle of interest from the order domain resampled signal. Finally, the Fourier transform is computed and the amplitude of the component of interest retained as a result from the current iteration. A data sample is hence obtained after the completion of the Monte Carlo run, from which the distribution of the population of the synchronous amplitude of interest can be inferred. The obtained distribution is then compared to the distribution obtained from the model in Eq. (23) and its exact version accounting for the cycles splitting. For the computed order tracking, piecewise linear approximation of the angle-time law is used as described in Section 2. In order to simplify the comparisons, high ratio of sampling frequency to reference frequency in time domain and spline interpolation to order domain are used for resampling the vibration signal. Such a choice guarantees that at most negligible attenuation is introduced from interpolation and allows reproducing the isolated effect of pulse uncertainty. Also, care is taken to using enough points for the angular domain resampling grid [42]. The jitter error is simulated by adding uniformly distributed random variables to the exact zeros coming from the simulated keyphasor signal at reference trigger frequency.

\subsubsection{Single harmonic signal}

In order to verify the numerical model, first a signal consisting of a single (complex) harmonic component is simulated. This case is interesting in order to understand the extent of amplitude attenuation in the averaged signal which is due to the jitter under different conditions. Different cases for ratio, number of revolutions and uncertainty extent are considered. The discrete signal is generated and its distribution estimated according to the parameters in Table 1 , whereas the parameters for the model-generated results are given in Tables 2, and 3 summarizes the considered cases for different combinations of ratio,

Table 1

Parameters for the generation of the single harmonic signal and its computed order tracked version from a discrete time-series simulating a vibration acquisition.

\begin{tabular}{lll}
\hline Parameter & Description [dimension] & Value \\
\hline$N_{\text {iter }}$ & Number of Monte Carlo iterations [-] \\
$F_{S}$ & Sampling frequency [Hz] & 1 E4 \\
$S P R$ & Angular samples per revolution [-] & 16384 \\
$f^{*}$ & Shaft of interest rotation frequency (fundamental frequency) [Hz] & 256 \\
$f_{H}$ & Frequency of the single-harmonic excitation [Hz] & 16 \\
$\gamma$ & Order of interest (in multiples of fundamental order) $[-]$ \\
$a$ & Amplitude of the harmonic excitation [-] & 1 \\
\hline
\end{tabular}


Table 2

Parameters for the simulation of the single harmonic signal amplitude distribution from the numerical models.

\begin{tabular}{lll}
\hline Parameter & Description [dimension] & Value \\
\hline$\left[0.5\right.$ ex] $N_{\text {iter }}$ & Number of Monte Carlo iterations [-] & $1 \mathrm{E} 6$ \\
$\Theta$ & Shaft of interest angular period [-] & 1 \\
$\lambda$ & Linear slope coefficient in the time-angle law [-] & 1 \\
$\gamma^{*}$ & Order of the sinusoidal excitation (in multiples of fundamental order $1 / \Theta)[-]$ & 1 \\
$\gamma$ & Order of interest (in multiples of fundamental order) [-] & 1 \\
$a$ & Amplitude of the harmonic excitation [-] & 1 \\
\hline
\end{tabular}

Table 3

Combinations of the studied parameters (Number of trigger revolutions, ratio and pulse time uncertainty extent as a fraction of the fundamental cycle frequency) presented for the numerical model validation.

\begin{tabular}{llll}
\hline Simulation number & $N_{t}$ & $r$ & $\Delta$ \\
\hline 1 & 40 & 1 & 0.2 \\
2 & 60 & 1 & 0.2 \\
3 & 80 & 1 & 0.2 \\
4 & 100 & 1 & 0.2 \\
5 & 40 & 2.286 & 0.2 \\
6 & 60 & 2.286 & 0.2 \\
7 & 80 & 2.286 & 0.2 \\
8 & 100 & 2.286 & 0.2 \\
9 & 100 & 2.286 & 0.1 \\
10 & 100 & 2.286 & 0.3 \\
11 & 100 & 2.286 & 0.4 \\
12 & 100 & 75.27 & 0.2 \\
\hline
\end{tabular}

number of revolutions and uncertainty extent. The computational burden associated with the discrete signal generation and order tracking limited the number of Monte Carlo runs used for the estimate. Estimation error can be expected to be higher for the tails of the distribution. Although error bars on quantiles estimation are not reported in the results, it was verified that the number of iterations allows for obtaining, far from the tails, sufficiently consistent distribution samples from the population also in the worst case of high values of the uncertainty.

Fig. 4a shows the cumulative distribution functions of the amplitude of the synchronous component in the signal as obtained from the synchronous average of the generated discrete signal using computed order tracking and from the exact numerical model, for different values of the number of trigger shaft revolutions $N_{t}$ and a single value of the ratio $r$. The results from the models and those from the computed order tracking numerical simulations agree very well, as the curves are hardly distinguishable. In this case, since the ratio $r$ is integer, the approximate model of Eq. (26) coincides with the exact model.

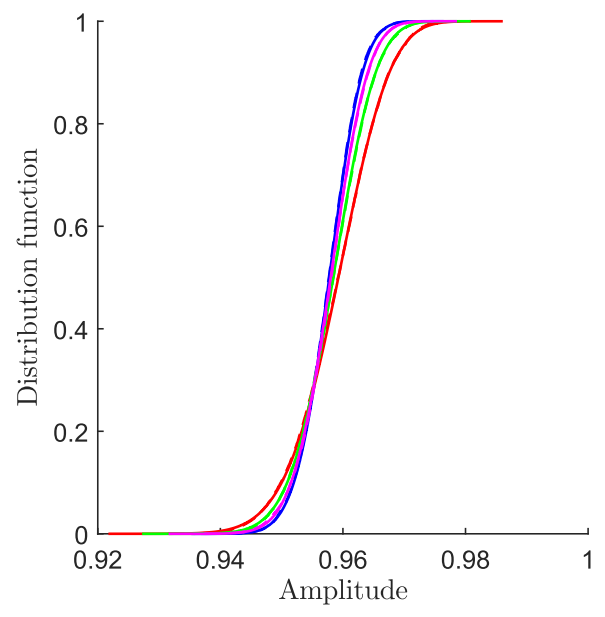

(a)

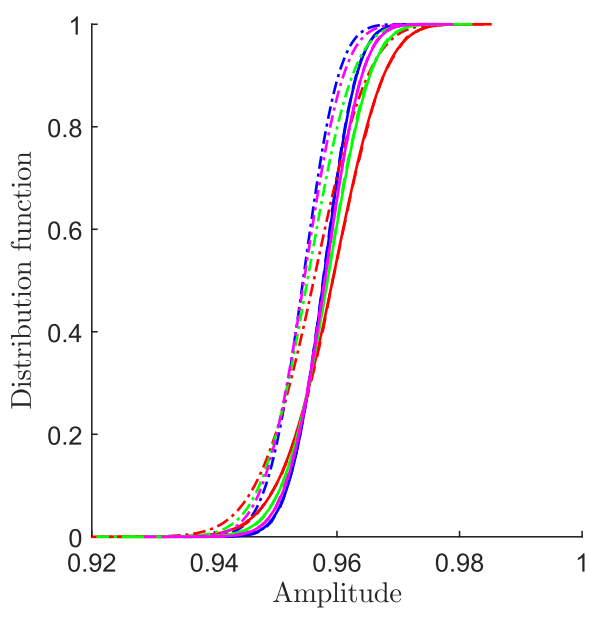

(b)

Fig. 4. Amplitude distribution functions for $\Delta=0.2$ varying $N_{t}$, obtained from the exact, approximated model and from the computed order tracking of a discrete signal. Continous lines: CDFs obtained from the model; dash-dotted lines: CDFs obtained from the approximated model; dashed lines: CDFs obtained from the order tracking simulations. Red: $N_{t}=40$; green: $N_{t}=60$; purple: $N_{t}=80$; blue: $N_{t}=100-$ a) $r=1$; b) $r=2.286$. (For interpretation of the references to colour in this figure legend, the reader is referred to the web version of this article.) 
Increasing the number of revolutions has the main effect of decreasing the dispersion of the results. Fig. 4b shows the same comparison for a non-integer value of the ratio $r$. Also in this case, the results from the exact model and from the computed order tracking simulations present an excellent agreement. However, small deviations are found in the results from the approximated model, attributable to the cycle assignment approximation. For the same value of the ratio $r$, Fig. 5 shows the cumulative distribution functions for different values of the uncertainty extent $\Delta$. An increased value of uncertainty on the time pulses determines an increased dispersion of the estimates, together with an attenuation of the mean value obtained for the amplitude of the synchronous component of interest. The approximated model's accuracy is lower as the uncertainty extent $\Delta$ is increased. Finally, in Fig. 6 the cumulative distribution functions are compared for different values of the ratio, holding the same uncertainty extent and number of revolutions. It can be observed that for the single harmonic case, varying the ratio does not result in significant changes of the distribution. For the high value of the ratio, the agreement of the results from the approximated numerical model increases due to reduced weight of the approximated cycles in the summations.

\subsubsection{Multi-harmonic signal}

In a general setting, it is interesting to understand how different synchronous orders influence each other for the reasons explained in Section 3. In this set of simulations, a signal composed of the sum of two (complex) harmonic excitations is simulated. The purpose is to show the adequacy of the model to simulate the results of a multi-harmonic excitation. The new parameters $r_{h a}$ and $r_{h f}$ are introduced, indicating respectively the ratio of the amplitude of the second harmonic component to that of the first harmonic component and the ratio of the frequency of the second harmonic component to that of the first one. The cumulative distribution functions of the amplitude of the fundamental component after averaging the multi-harmonic signal are investigated. For a fixed integer value of the shaft speed to trigger speed ratio $r$, number of revolutions $N_{t}$, amplitude ratio $r_{h a}$ and uncertainty extent, the results for different values of $r_{h f}$ are shown in Fig. 7a. The results for the non-integer ratio $r$ value are in a good agreement, with the approximate model showing significant deviation, indicating that it is not suitable for the multi-harmonic analysis due to the inability of properly coping with interactions between harmonics. It can be observed that the distributions are very similar to those of the single harmonic case, indicating a small influence from the additional synchronous component. The main difference is in the increased dispersion due to mutual orders interactions, which is higher when the interacting order is closer. Fig. $7 \mathrm{~b}$ reports the results of the simulation obtained using an integer value of the ratio. In this case, the approximated model equations are equivalent to the exact numerical model. The obtained distributions are significantly different from those of the single harmonic case also for what concerns the average attenuation, indicating high interaction between the two considered harmonics. This result is explained by the fact that for integer ratios, the amplitude at the considered order $\gamma$ originating from the excitation $r_{h f} \gamma^{*}$ is consistently higher than for non-integer ratios, due to the averaged excitations being in phase on average.

\subsection{Numerical validation of the analytical approximation}

The approximation derived in Section 3.3 allows to quickly study the attenuation and the variance of the synchronous components introduced from the random jitter error in the synchronous average estimates. The aim of the current section is to validate the derived expressions for the synchronous amplitude distribution through comparison with the Monte Carlo

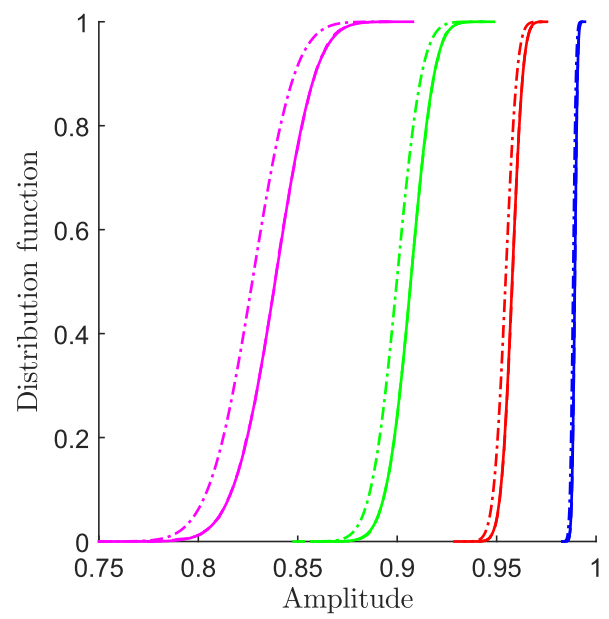

Fig. 5. Amplitude distribution functions for non-integer ratio and $N_{t}=100$ varying $\Delta$, obtained from the exact, approximated model and from the computed order tracking of a discrete signal. Continous lines: CDFs obtained from the model; dash-dotted lines: CDFs obtained from the approximated model; dashed lines: CDFs obtained from the order tracking simulations. Blue: $\Delta=0.1$; red: $\Delta=0.2$; green: $\Delta=0.3$; purple: $\Delta=0.4$. (For interpretation of the references to colour in this figure legend, the reader is referred to the web version of this article.) 


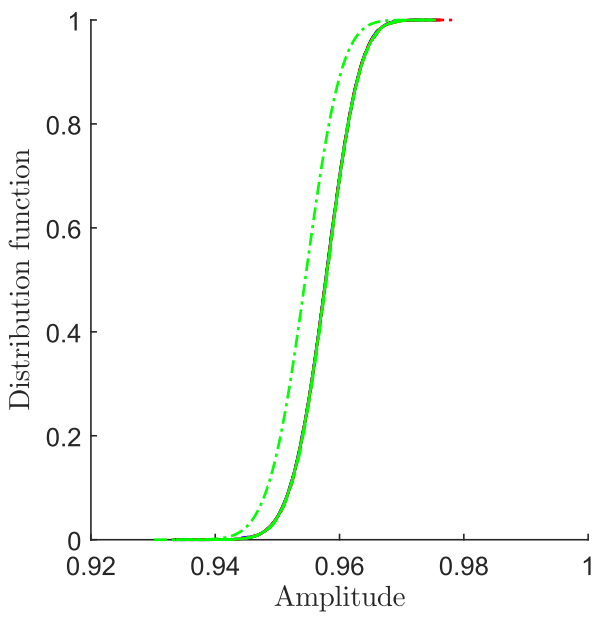

Fig. 6. Amplitude distribution functions for $N_{t}=100$ and $\Delta=0.2$ varying the ratio $r$, obtained from the exact, approximated model and from the computed order tracking of a discrete signal. Continous lines: CDFs obtained from the model; dash-dotted lines: CDFs obtained from the approximated model; dashed lines: CDFs obtained from the order tracking simulations. Blue: $r=1$; green: $r=2.286$; red: $r=75.27$. (For interpretation of the references to colour in this figure legend, the reader is referred to the web version of this article.)

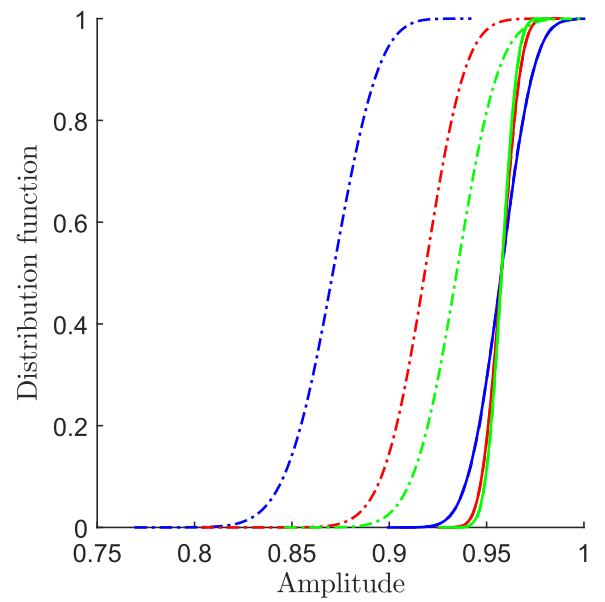

(a)

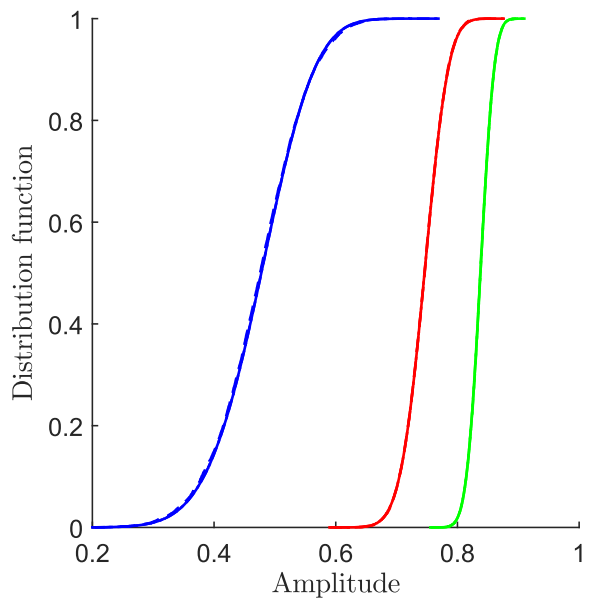

(b)

Fig. 7. Amplitude distribution functions for $N_{t}=100, \Delta=0.2$ and $r_{h a}=20$, varying $r_{h f}$ in the multi-harmonic case, obtained from the exact, approximated model and from the computed order tracking of a discrete signal. Continous lines: CDFs obtained from the model; dash-dotted lines: CDFs obtained from the approximated model; dashed lines: CDFs obtained from the order tracking simulations. Blue: $r_{h f}=2$; red: $r_{h f}=3$; green: $r_{h f}=4-(\mathrm{a}) r=2.286$; $(\mathrm{b})$ $r=1$. (For interpretation of the references to colour in this figure legend, the reader is referred to the web version of this article.)

estimates of the same distribution obtained from the numerical model equations. Since the ratio $r$ was shown not to be influential in the single harmonic case, the simplified integer ratio case with $r=1$ can be studied as representative. $\mathrm{n}$ (see Fig. 3 for the reference values). The solutions obtained using the truncated series expansions hence provide an accurate approximation of the quantities of interest.

In Fig. 8, the cumulative distribution functions of the squared synchronous amplitude value obtained from the approximated analytical solution are compared to the cumulative distribution functions obtained through the Monte Carlo runs ( $1 e 5$ iterations) from the exact numerical model for a number of trigger periods $N_{t}=20$ (left) and $N_{t}=100$ (right), for values of the uncertainty extent $\Delta=\{0.1,0.2,0.3,0.4\}$. Squared values are used to simplify the comparisons by making possible to use directly Eq. (58) to plot the analytical solutions. The good agreement confirms that the analytical approximation can be used to study effectively the statistics of the extracted synchronous components amplitude in the case of a single harmonic. As expected, for the high values of $\Delta$, the approximation results in a slight underestimation of the expected value. The small dispersion of the simulated results around the approximated solution confirms that the Monte Carlo simulations from the numerical model provide accurate statistics for the considered cases. By analyzing the cumulative distribution functions, it is observed that the values are mostly distributed around the mean, with no significant tails and no significant skewness. 


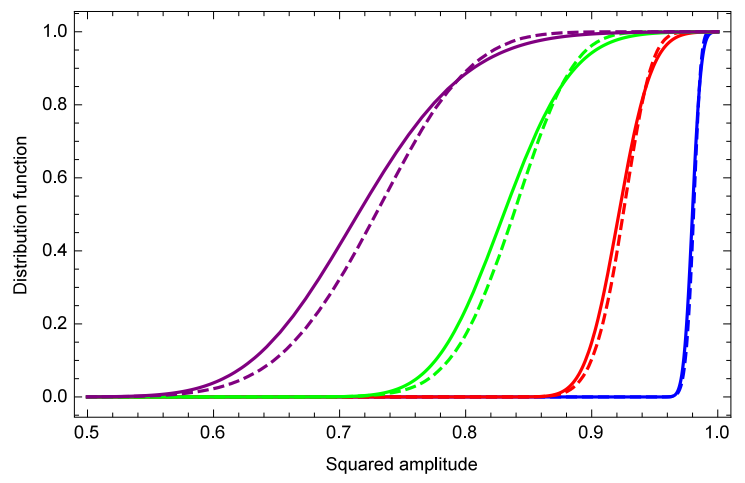

(a)

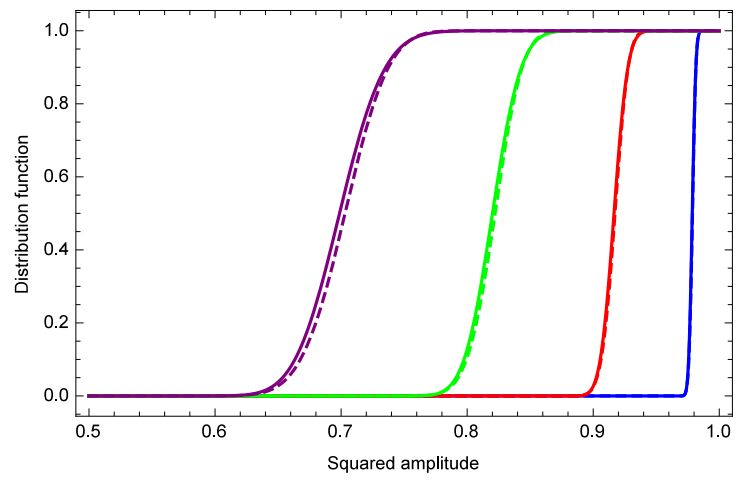

(b)

Fig. 8. Comparison of approximate analytical CDFs and CDFs simulated from the exact numerical model for different values of $\Delta$ and $N_{t}-$ Continous lines: theoretical predictions; Dashed lines: CDFs estimated from Monte Carlo simulation using the exact numerical model. Blue, red, green and purple used respectively for $\Delta=\{0.1,0.2,0.3,0.4\}-$ (a) $N_{t}=20$; (b) $N_{t}=100$. (For interpretation of the references to colour in this figure legend, the reader is referred to the web version of this article.)

\subsection{Parametric study}

The approximate analytical solution can be used to assess the effect of each parameter on the statistics of the squared amplitude values. In this section, only the first two cumulants are studied. Fig. 9 shows the expected value (left) and the variance (right) of the squared amplitude of a synchronous order as a function of the number of averages $N_{t}$ and the uncertainty extent $\Delta$, computed with the ratio parameter $r$ set to unity. From the figures, one has an immediate estimate of how the normalized uncertainty influences the synchronous average estimates for the synchronous component of interest. As from Eq. (47), the effect of the number of averages on the expected value is small and rapidly vanishes for a high number of averages. Conversely, increasing the number of averages helps reducing the variance of the estimate. It can be observed that for a number of averages typical in a monitoring system, ranging approximatively from 20 to 200, depending on the machine rotational speed and acquisition parameters, the gradient is still steep, meaning that a small increase in the number of averages can significantly improve the estimation variance. The uncertainty extent $\Delta$ influences both the expected value and the variance, resulting respectively in increasingly significant attenuation bias and variance of the estimates. Fig. 10 shows the effect of additive noise on the same cumulants, for a fixed value of $\Delta=0.2$ and varying the number of averages $N_{t}$. The relative change of the variance due to the noise is higher than that of the expected value. As one could expect from the theoretical discussion, it can be observed that increasing the number of averages has the effect of significantly reducing the impact of the noise on the estimates. Fig. 11 shows the cumulants as a function of the noise variance $\sigma_{n}^{2}$ and of the uncertainty extent $\Delta$, for a small number of averages $N_{t}=20$ (top row) and for a higher number of averages $N_{t}=100$ (bottom row). While the effect of the noise on the first cumulant stays almost negligible for any value of $\Delta$, its effect on the second cumulant is significant, especially for smaller number of averages. Henceforth, the main effect of random additive noise on the estimates under uncertain pulse times is that of increasing the estimation variance.

\section{Experimental validation}

\subsection{Seeded fault test on type 1 helicopter gearbox}

In this section, data from a seeded fault test is used in order to first verify the previous results, and then to show the consequences of the pulse time uncertainty on the detection of a gear fault consisting of a chipped tooth and developed surface pitting.

\subsubsection{Experimental set-up}

The seeded fault test was performed with the original purpose of collecting vibration data to verify and refine the HUMS (Health and Usage Monitoring System) algorithms for fault detection and diagnosis. Vibration and shaft angular position measurements were recorded from accelerometers and a magnetic pick-up mounted on a full-scale type 1 helicopter gearbox. A schematics of the type 1 gearbox is given in Fig. 12. The position of the sensors used for the present analysis is indicated. Accelerometers are indicated with the identifier $A$ and the magnetic pick-up with the identifier $M$. The accelerometers were used to record vibration at different locations, whereas the magnetic pick-up provided a keyphasor signal consisting of six pulses per revolution of the intermediate gear to the tail drive output. Fig. 13 shows the gearbox used for the test and the test stand. 


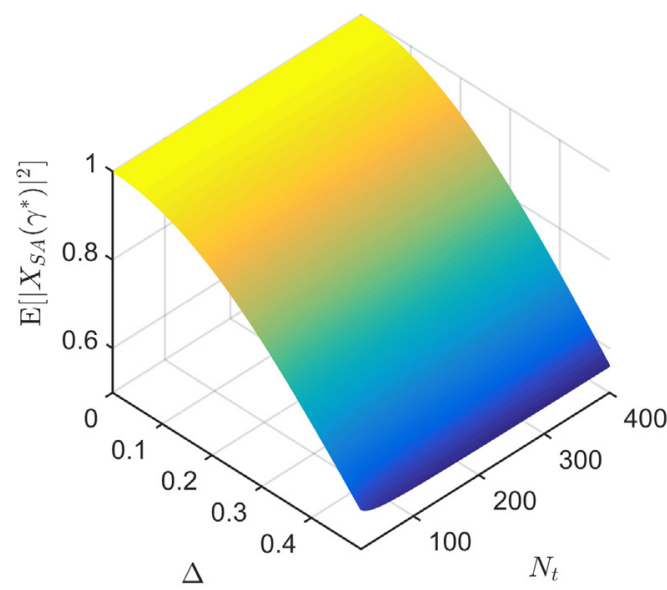

(a)

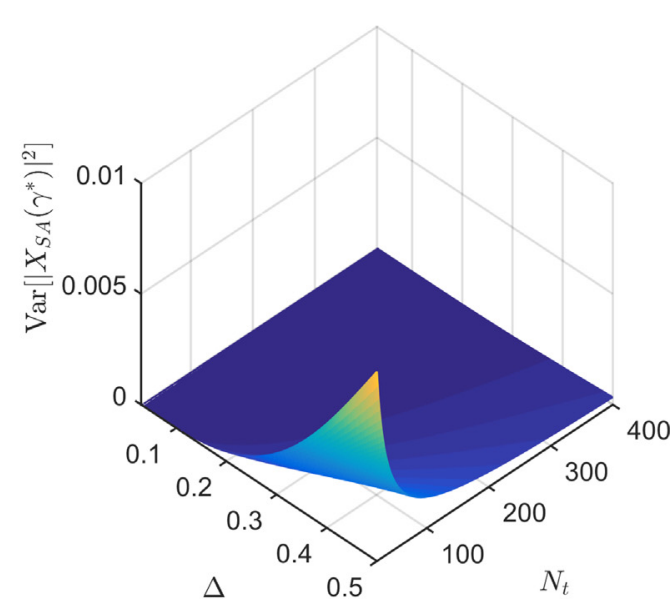

(b)

Fig. 9. First two cumulants of the squared amplitude of a synchronous order $\gamma^{*}$ for $r=1$, varying the number of averages $N_{t}$ and the uncertainty extent $\Delta-$ (a) Expected value; (b) Variance.

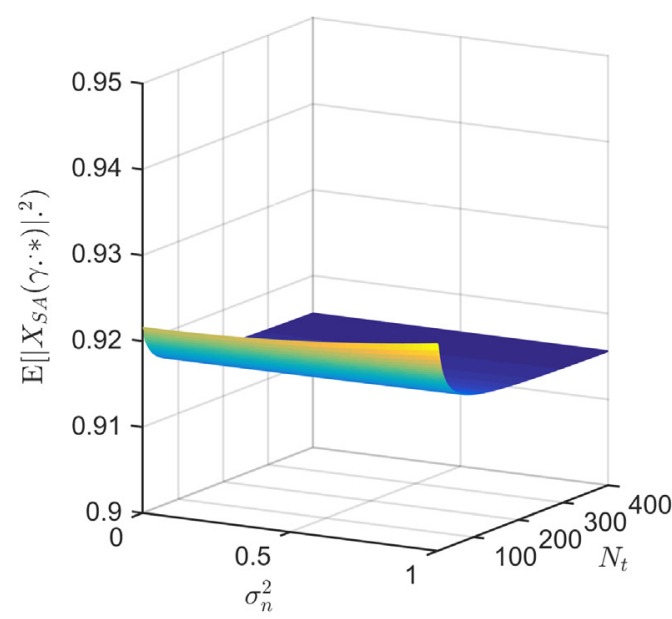

(a)

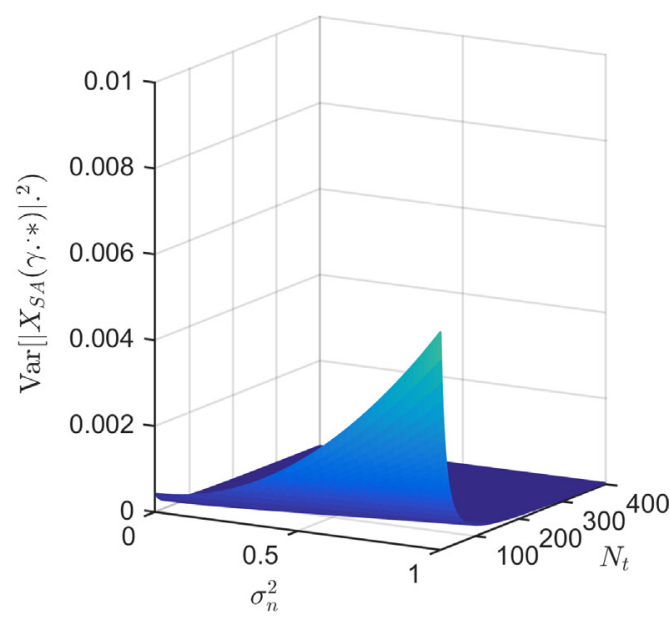

(b)

Fig. 10. First two cumulants of the squared amplitude of a synchronous order $\gamma^{*}$ for $r=1$ and $\Delta=0.2$, varying the number of averages $N_{t}$ and the noise variance $\sigma_{n}^{2}-($ a) Expected value; (b) Variance.

The test included six distinct thirty-minutes runs involving different operating conditions of the gearbox. Different combinations of the transmitted torque levels were tested, including one engine inoperative (OEI) conditions, obtained by transmitting torque to the gearbox only from one of the engines. Data from the first, third and fifth run was acquired in healthy conditions. During the sixth run, a faulty input pinion was introduced within the left-side input assembly, shown in Fig. 14. The second and fourth run involved different seeded faults, not discussed in this paper. Each test ran about one hour and thirty minutes, during which six different operating conditions were imposed. Namely, after a stabilization phase with low input torque, measurements were taken with an average torque level $\mathrm{T} 1 \mathrm{from}$ both the engines; then the input torque was increased by sixty percent up to level T2. OEI states for left and then right engine with the running engine operating at an intermediate torque level T3 were then measured. Finally, measurements were taken again with both engines providing torque level T1. Acquisitions were synchronously performed each five minutes and include vibration data from the accelerometers, and keyphasor data from the magnetic pick-up. For data acquisition, a hardware with comparable characteristics to those required for an operating helicopter was employed. However, higher sampling frequencies than normal were used for the test, since data storage constraints in laboratory are not as strict as those on board. Acceleration signals were sampled at $93750 \mathrm{~Hz}$, whereas keyphasors signals were made available both with $f_{K P 1}=93750 \mathrm{~Hz}$ sampling frequency and with $f_{K P 2}=23438 \mathrm{~Hz}$ sampling frequency. The hardware returns keyphasor signals as square waves with a change in the polarization indicating each time that the signal has reached the trigger level. 


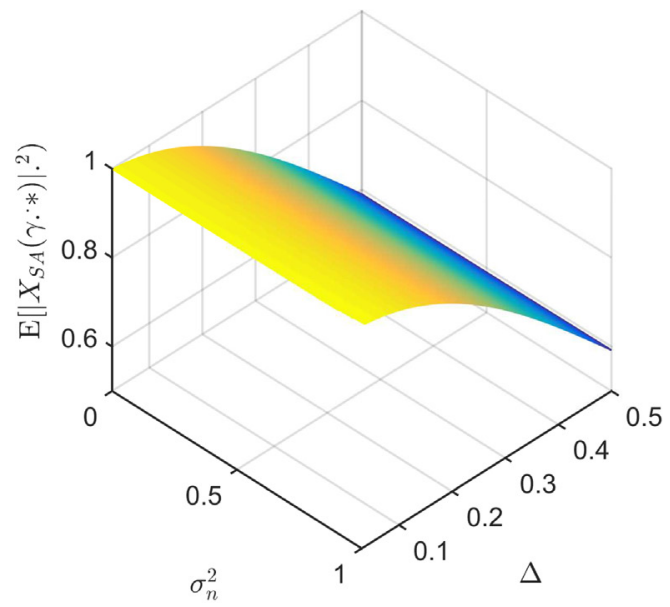

(a)

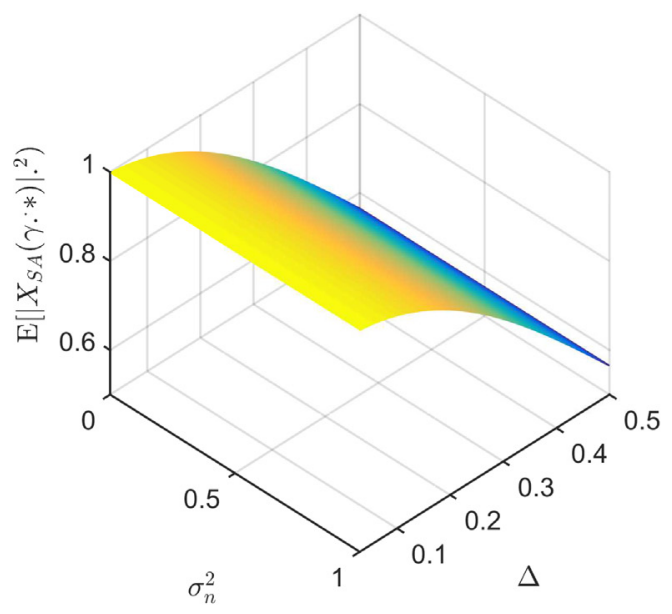

(c)

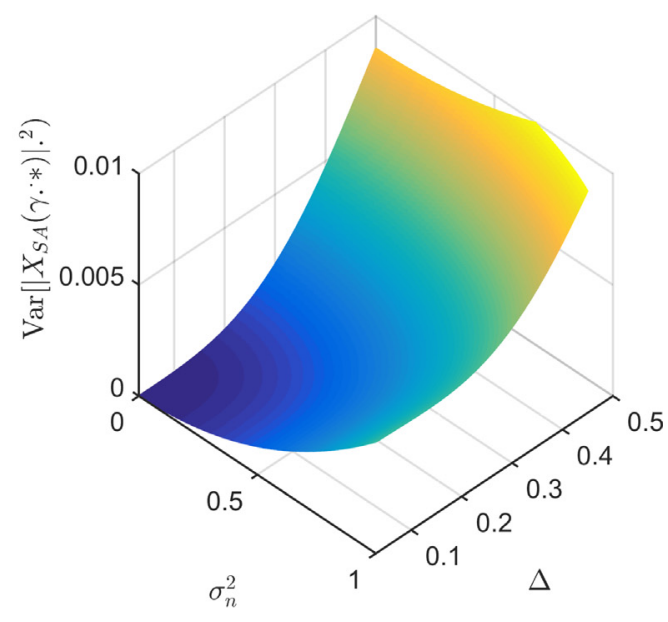

(b)

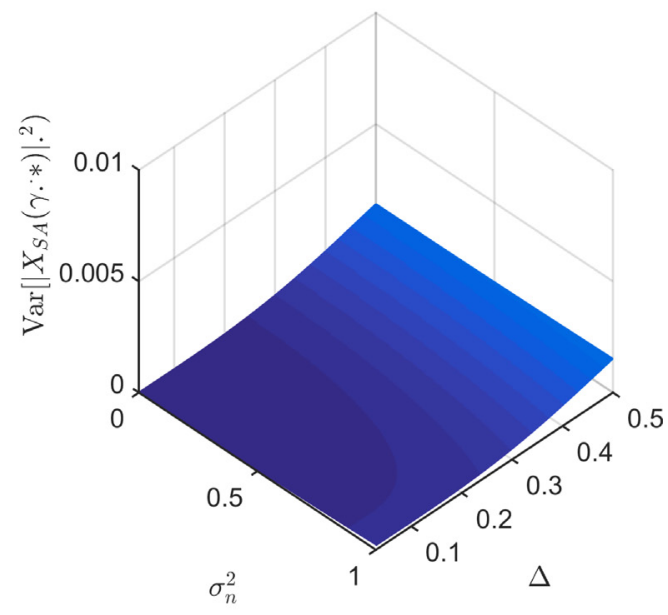

(d)

Fig. 11. First two cumulants of the squared amplitude of a synchronous order $\gamma^{*}$ for $r=1$, varying the uncertainty extent $\Delta$ and the noise variance $\sigma_{n}^{2}-$ (a) Expected value, $N_{t}=20$; (b) Variance, $N_{t}=20$; (c) Expected value, $N_{t}=100$; (d) Variance, $N_{t}=100$.

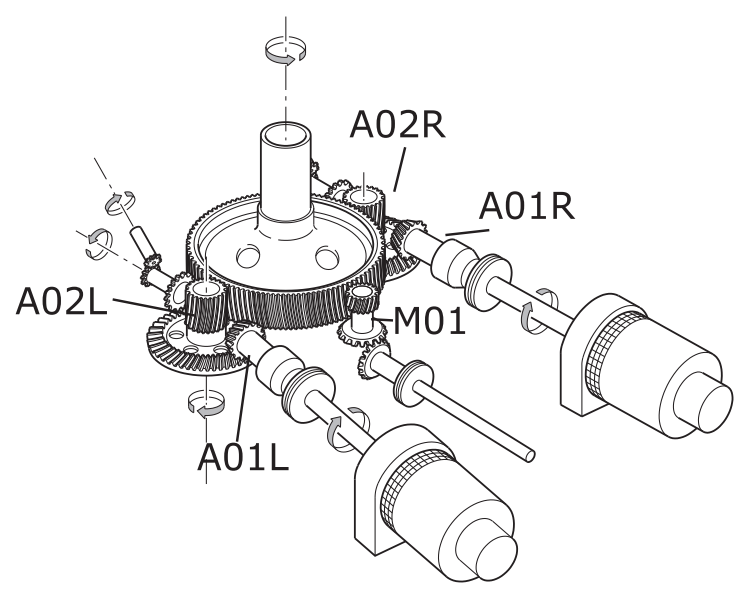

Fig. 12. Type 1 gearbox schematics, indicating the position of the sensors considered for the analysis. 


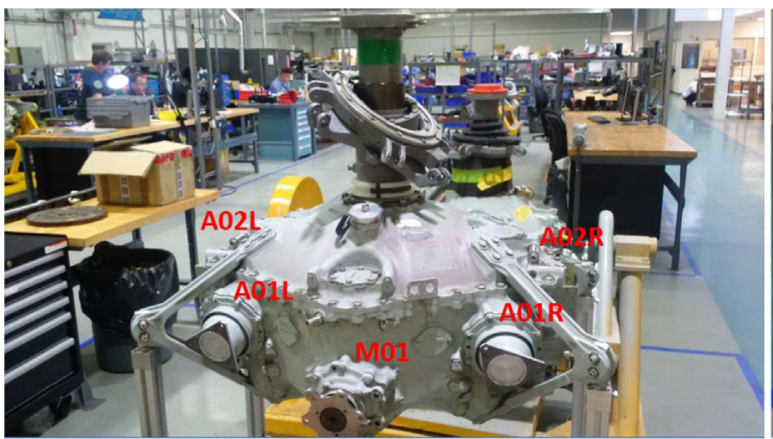

(a)

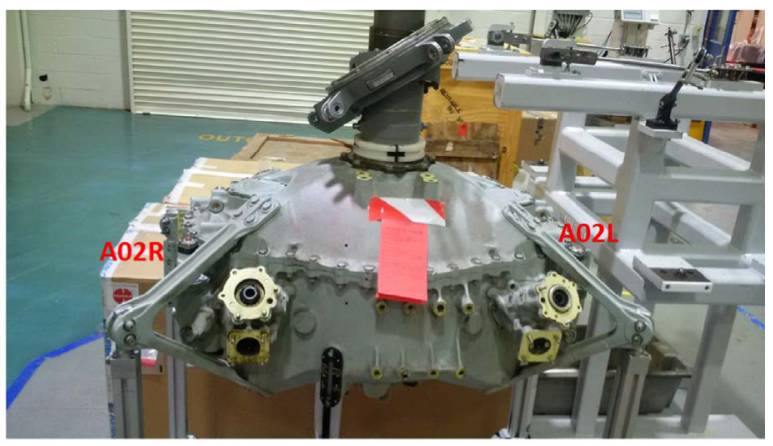

(b)

Fig. 13. The tested gearbox before the installation of the monitoring system. Sensor locations are indicated in red - (a) Front view; (b) rear view.

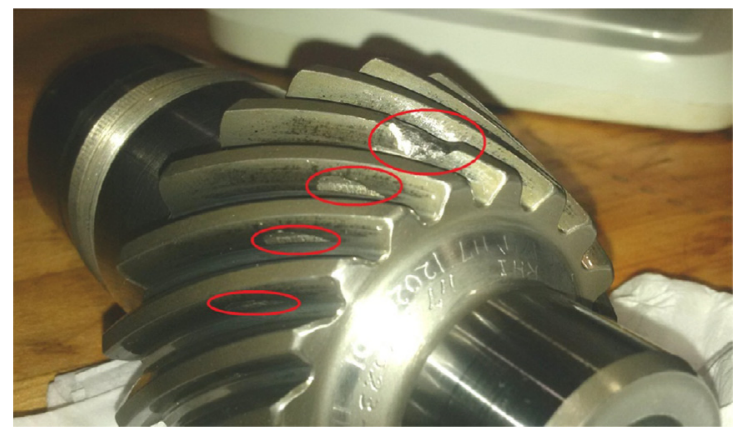

Fig. 14. Left input pinion with a chipped tooth and pitted surface areas circled in red. (For interpretation of the references to colour in this figure legend, the reader is referred to the web version of this article.)

\subsubsection{Effect of keyphasor uncertainty: verification}

The aim of this section is to show that the modelling assumptions for the jitter effects are consistent for a gearbox running nominally at constant speed, including small fluctuations as described in Section (2). First, it is shown that a piecewise function for angle-time progression estimation is more robust to keyphasor uncertainty than the commonly used second-order approximation [35] or the spline curve-fitting [41]. Secondly, the predictions from the model developed in Section 3.2 are verified using the experimental data. In order to do that, for each available record the two keyphasor signals (low and high frequency) are used to order track the shaft angle using the piecewise linear approximation, transforming then the vibration signals into the angular domain according to the process outlined in Section 2.1 and summarized in ??. One pulse per revolution of the reference shaft is used, discarding the other five pulses. This choice is motivated from the hypothesis of small, slow speed fluctuations and allows to avoid the additional errors introduced from non-uniform angular spacing. Synchronous averages are performed as from Section 2.1 with the aim of extracting a fundamental cycle of the input drive shaft. The shafts speed ratio between target and reference (trigger) shaft is computed using kinematics relations. The amplitude of the first and second gear mesh components of the input drive shaft is then exctracted from the synchronous average estimate for each sensor. Finally, the ratios between the gear mesh amplitude computed with the low sampling frequency keyphasor signal and that computed with the high sampling frequency keyphasor signal are calculated. Parameters for order tracking and synchronous average are given in Table 4.

By assuming that the only cause for deviation from unity is the higher uncertainty of the pulse times, the amplitude ratios calculated for each record can be used to estimate the distribution function of the attenuation resulting from the increased jitter level. The distribution functions of the amplitude ratio obtained for each sensor are then compared to the theoretical predictions from the model. The process is repeated using the second-order and the spline approximations in the order tracking step, and the resulting distribution functions are also reported. The first and second harmonics of the gear mesh was chosen for two main advantages: on one hand, their frequency is sufficiently low with respect to the vibration signal sampling frequency, and therefore the interpolation attenuation due to the spline resampling (second step of the order tracking procedure) is approximately constant and close to the unity within the average speed fluctuation range; on the other hand, the signal to noise ratio is high for those harmonics, allowing to obtain good estimates of the amplitude using the synchronous average procedure.

Fig. 15 shows an example of the frequency spectrum obtained through Welch's method for one of the vibration records from sensor A01R during Run 1 in T1 torque conditions. It can be observed that the amplitude of the first gear mesh 
Table 4

Parameters for order tracking and synchronous average using bench data.

\begin{tabular}{ll}
\hline Parameter & Description [dimension] \\
\hline$N_{t}$ & Number of revolutions of the reference shaft [-] \\
$r$ & Ratio of the target shaft speed to the reference shaft speed [-] \\
$S P R$ & Angular samples per revolution [-] \\
$\gamma_{1}$ & Order of the first gear mesh harmonic (in multiples of fundamental order) [-] \\
$\gamma_{2}$ & Order of the second gear mesh harmonic (in multiples of fundamental order) [-] \\
$f_{K P 1}$ & Sampling frequency of the fast keyphasor signal [Hz] \\
$f_{K P 2}$ & Sampling frequency of the slow keyphasor signal [Hz] \\
\hline
\end{tabular}

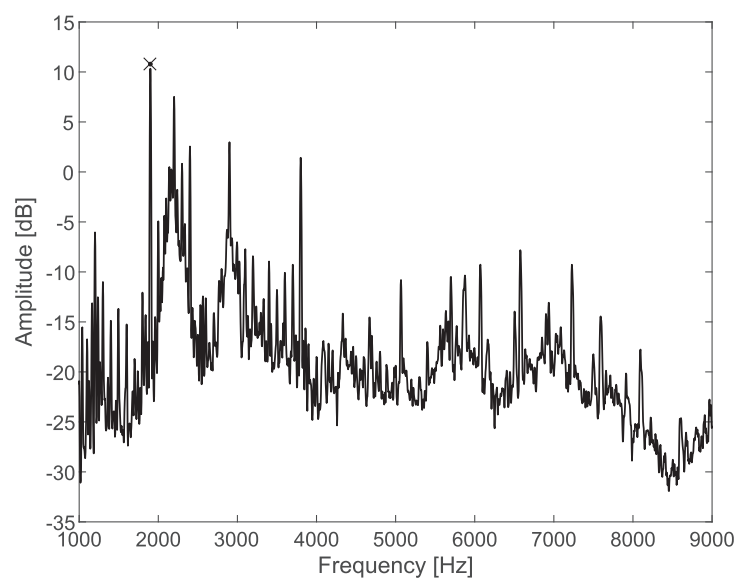

Fig. 15. Spectrum of a 2 s fault-free vibration signal recorded in during run 1 from A01R accelerometer with a sampling frequency of 93750 Hz (estimated using Hanning window and 16 averages with 50\% overlap). Scale is restricted to the range [1-9] kHz for visualization purposes. Black cross: input pinion first harmonic of the gear mesh frequency.

harmonic, located at around $1900 \mathrm{~Hz}$ (marked with a black cross in the figure), is high compared to the background noise. An additional noise-like contribution can be expected from the close family of harmonics located in the $2-3 \mathrm{kHz}$ range. Similarly, the second harmonic amplitude is comparatively high with respect to the background noise. The spectral smearing effect due to the small speed fluctuations is also clear from the figure, especially for the high-frequency harmonics. Fig. 16a and b show the distribution functions of the first and second gear mesh harmonics amplitude ratio, obtained for the signals from the four available accelerometers as formerly explained and using the piecewise linear order tracking. Theoretical predictions from the model, obtained using Monte Carlo simulations, are shown as the black continuous lines in the same plots. For this experiment, the approximate analytical solutions for the distribution functions could not be directly used, as the statistics had to be computed from the ratio of the two distributions under two different values of uncertainty. The average attenuation is predicted very well. However, the experimental ratios suffer from higher dispersion due to the residual additive noise (see Section 3.3.1). It is observed that the distribution functions from those sensor mounted closer to the meshing gears, i.e. A01L and A01R, present less dispersion and match better the noise-free predictions. In fact, the signal to noise ratio is higher for the sensors positioned close to the meshing gears. A better agreement is found for the second gear mesh harmonic, confirming that the signal to noise ratio after synchronous averaging is higher for that harmonic, at least when measuring close to the meshing gears. Fig. 16c, and e and Fig. 16d, and f, show the same distribution functions obtained respectively with a piecewise second-order polynomial approximation and with a spline interpolation for the angle-time law.

It can be observed that the amplitude ratio, representing the inverse of the attenuation due to jitter of the reference keyphasor pulses, is higher for the piecewise linear approximation at all sensor locations for both the harmonics, confirming that when the dominant error contribution comes from jitter uncertainty, a simple but adequate approximation law for the angle-time relation is a better choice.

\subsubsection{Effect of keyphasor uncertainty on gear fault detection using residual signal}

The aim of this section is to show the impact of keyphasor pulse uncertainty on the detection of the gear fault using the residual signal [48]. The residual signal can be obtained from the synchronous average signal by removing the gearmesh harmonics, representing the average mesh cycle. It was shown to be effective for gear local defects diagnosis [49]. Compared to other popular techniques such as amplitude or frequency demodulation [50], AR residual [51], or methods based on Spectral Kurtosis and Minimum Entropy Deconvolution [52,53], the synchronous average residual signal has the advantage of not requiring the specification of parameters such as the demodulation band, the model order, window or filter lengths. It 


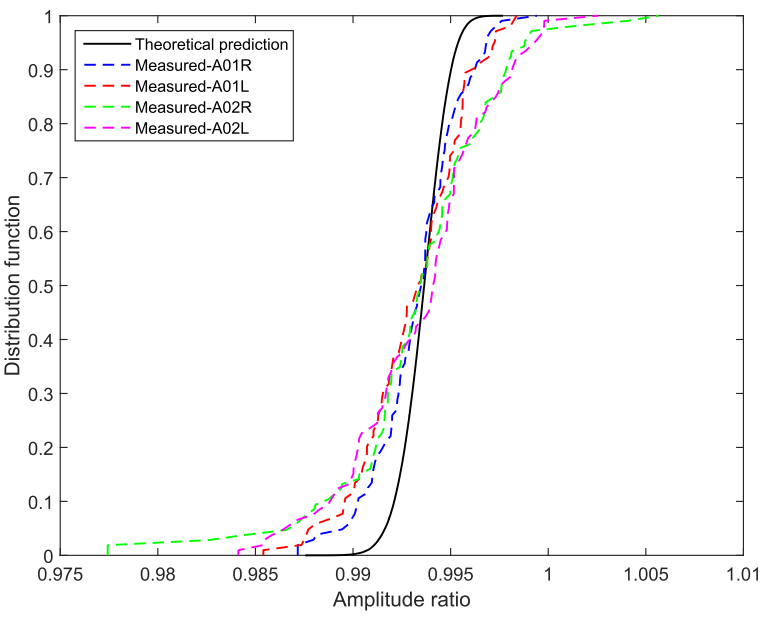

(a)

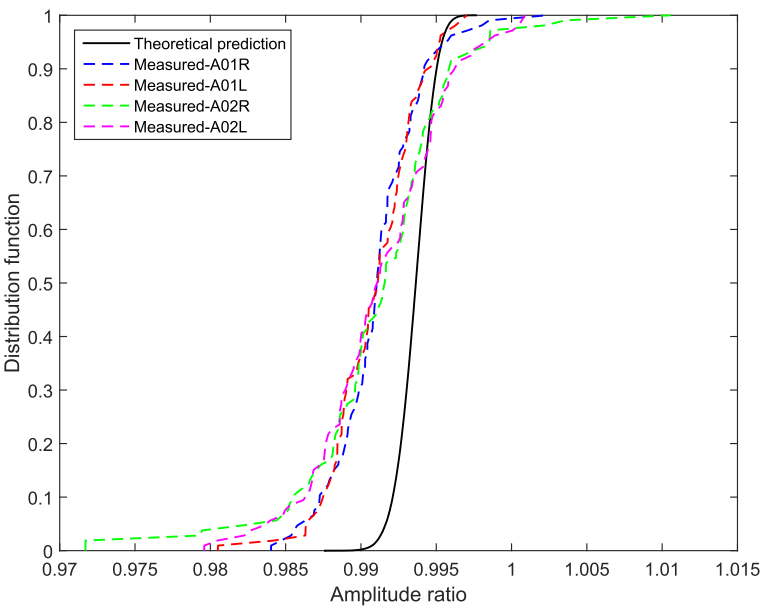

(c)

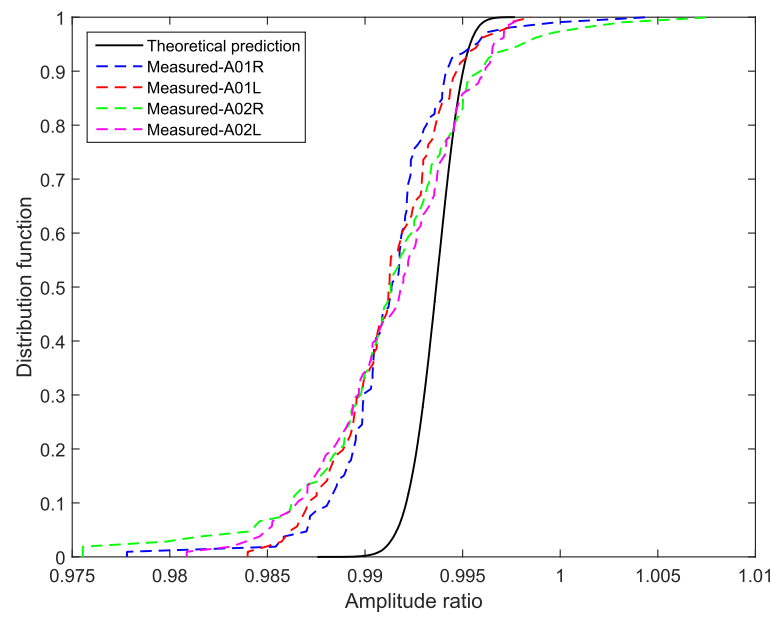

(e)

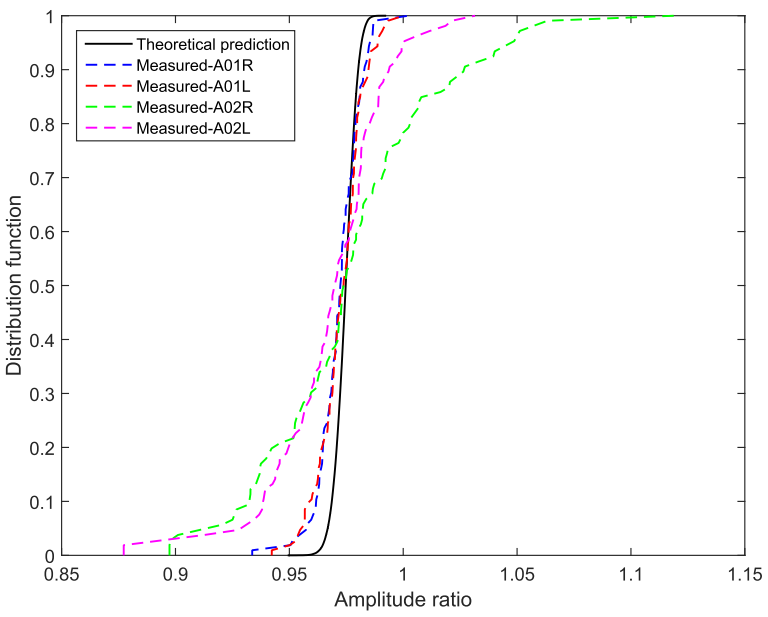

(b)

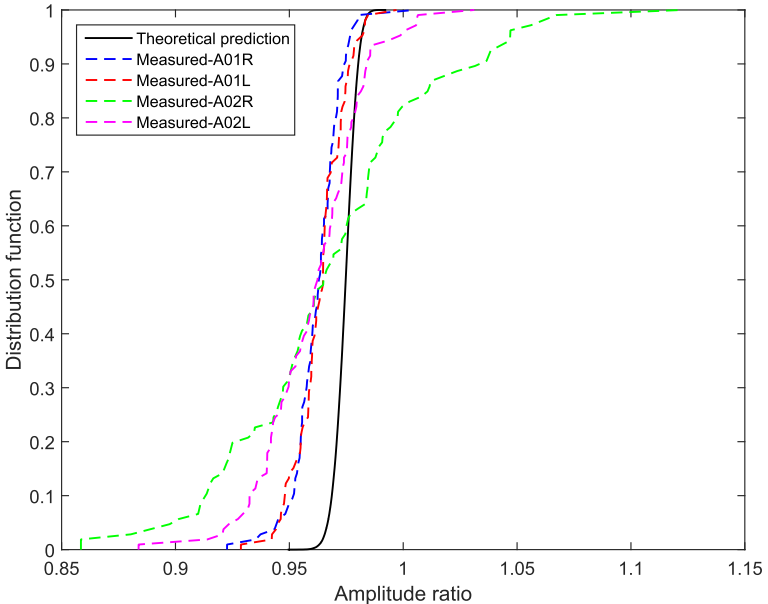

(d)

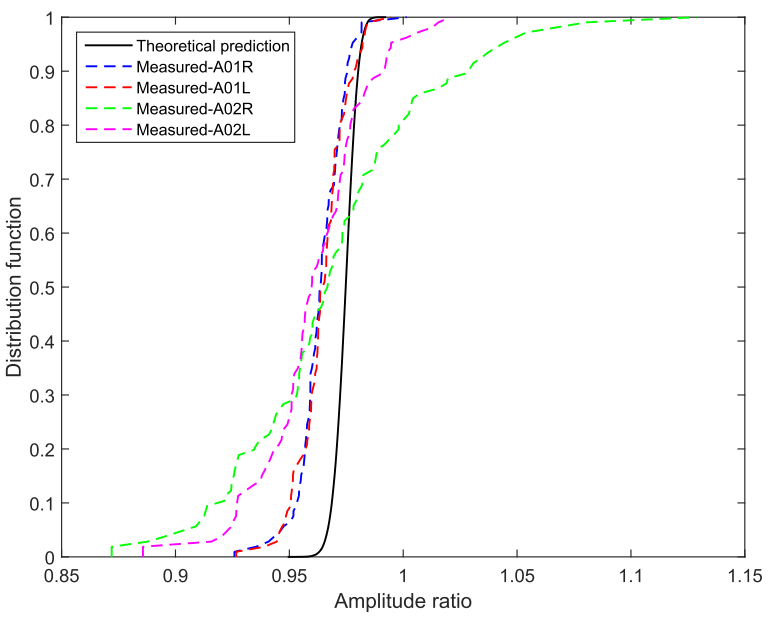

(f)

Fig. 16. Distribution function of the amplitude ratio parameter for the first (left) and second (right) gear mesh harmonics of the input drive shaft. Black: theoretical predictions; dashed blue: measurements from accelerometer A01R; dashed red: measurements from accelerometer A01L; dashed green: measurements from accelerometer A02R; dashed purple: measurements from accelerometer A02L - (a),(b) Piecewise linear order tracking model; (c),(d) piecewise second order polynomial order tracking model; (e),(f) spline interpolation order tracking model. (For interpretation of the references to colour in this figure legend, the reader is referred to the web version of this article.) 
can therefore easily be used in an automated detection framework without the need for extensive tuning, albeit generally suffering from lower performance in the detection with respect to the more sophisticated methods.

Typically, the kurtosis (fourth statistical cumulant) of the residual signal is a good indicator for local gear fault detection [49] and can be used as a practical indicator in a monitoring framework. A good estimate of the synchronous average is important for obtaining a representative residual. The keyphasor pulse uncertainty can compromise the fault detection by leading to a low-quality estimate of the synchronous average and, consequently, of the residual signal. Figs. 17a and b, show respectively the residual signal obtained for one acquisition during run 1 and during run 6 using sensor A02L. The local defect is visible at around 50 degrees within a cycle of the input drive shaft in Fig. $17 \mathrm{~b}$.

In order to simulate an operational setting, the vibration signals are re-sampled to sampling frequencies of $4687.5 \mathrm{~Hz}$ and $9375 \mathrm{~Hz}$, representing conditions in which the Nyquist bandwidth allows respectively to observe at most the first, and the second gearmesh frequency. Those scenarios are common for helicopters' monitoring systems. Signals from accelerometer A02L were taken, as less sensitive to operating conditions variations, supposedly because of the sensor being located further from the coupling of the input drive shaft with the engines. The kurtosis value of the residual signals is then computed for all the acquisitions available from runs 1,3 and 5 (healthy pinion), and for those acquisitions available from run 6 (faulty pinion). A first set of values is obtained using the most exact pulse times for signal synchronization, whereas a second set is obtained by adding a uniformly distributed error on such times. The extent of uncertainty for the uniformly distributed random variables is computed as to simulate a pulse arrival time error equivalent to the one that would be obtained by sampling the keyphasor at $4687.5 \mathrm{~Hz}$ and $9375 \mathrm{~Hz}$. Fifty simulations were performed with fifty different realizations of the uncertainty.

Fig. 18 shows a comparison between the values of residual kurtosis obtained using the most exact available pulse arrival times (reference indicated as $f_{k p 1}$ ) and those obtained by adding the random variables to the pulse arrival times (reference indicated as $f_{k p 2}$ ), for the case of a bandwidth limited to the first gear mesh harmonic. Fig. 19 shows the same comparison for the case of a bandwidth allowing to observe up to the second gear mesh harmonic. A good discrimination between damaged and healthy state can be performed on the basis of the residual kurtosis indicator when using the exact pulse times. From both the figures, it is clear that the pulse times uncertainty has a detrimental effect on the distinguishability of the damaged state. Even though the loss of performance is not as severe as to impede the detection of the considered fault, especially when the keyphasor modified frequency allows up to the second gear mesh, it may result in a delayed detection within an operational framework. It is therefore important to keep into account the need for a precise reference keyphasor signal for monitoring applications.

\subsection{Flight data analysis from type 2 fleet}

In the following, the results obtained in the previous sections are verified using real flight data acquired in a variety of operating conditions from fourteen different helicopters. The aim of this investigation is to assess that the model under the derived assumptions allows representing an operating scenario.

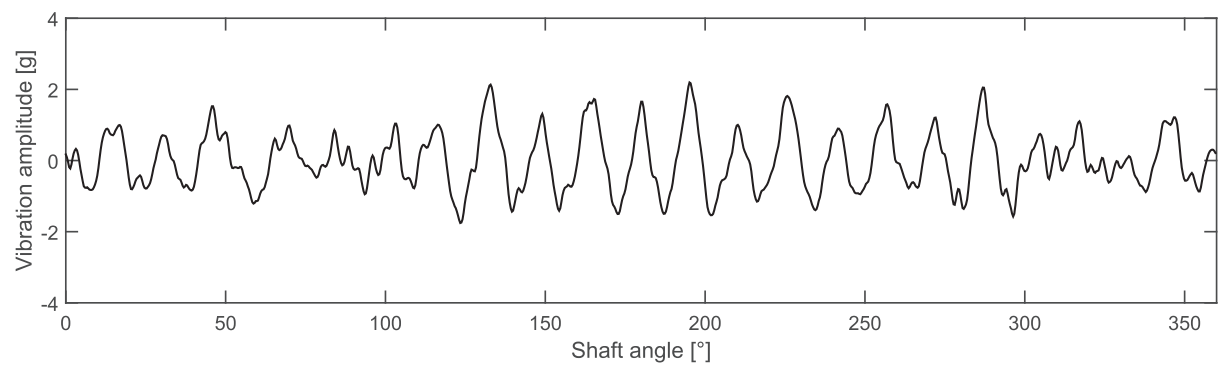

(a)

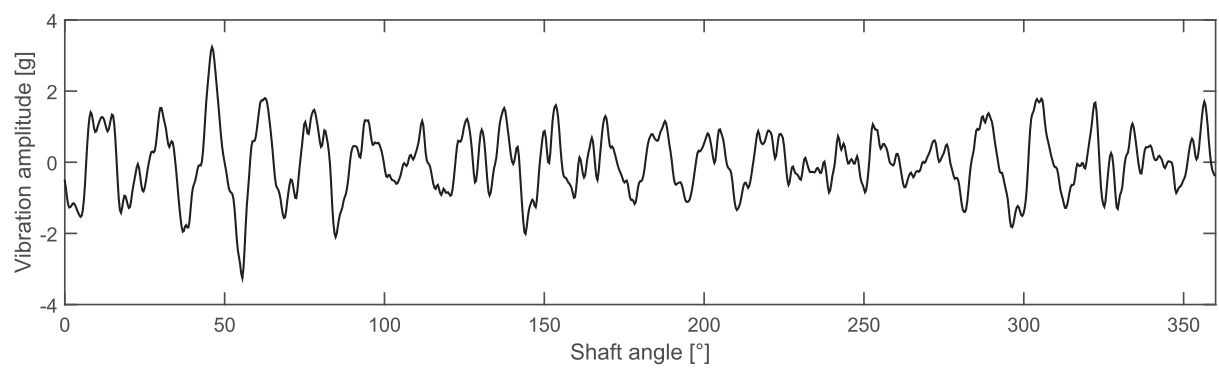

(b)

Fig. 17. Residual signals for one revolution of the input pinion obtained from accelerometer A01L - (a) Healthy signal from run 1 : Kurtosis value $=2.54$; (b) Faulty signal from run 6: Kurtosis value $=3.42$. 

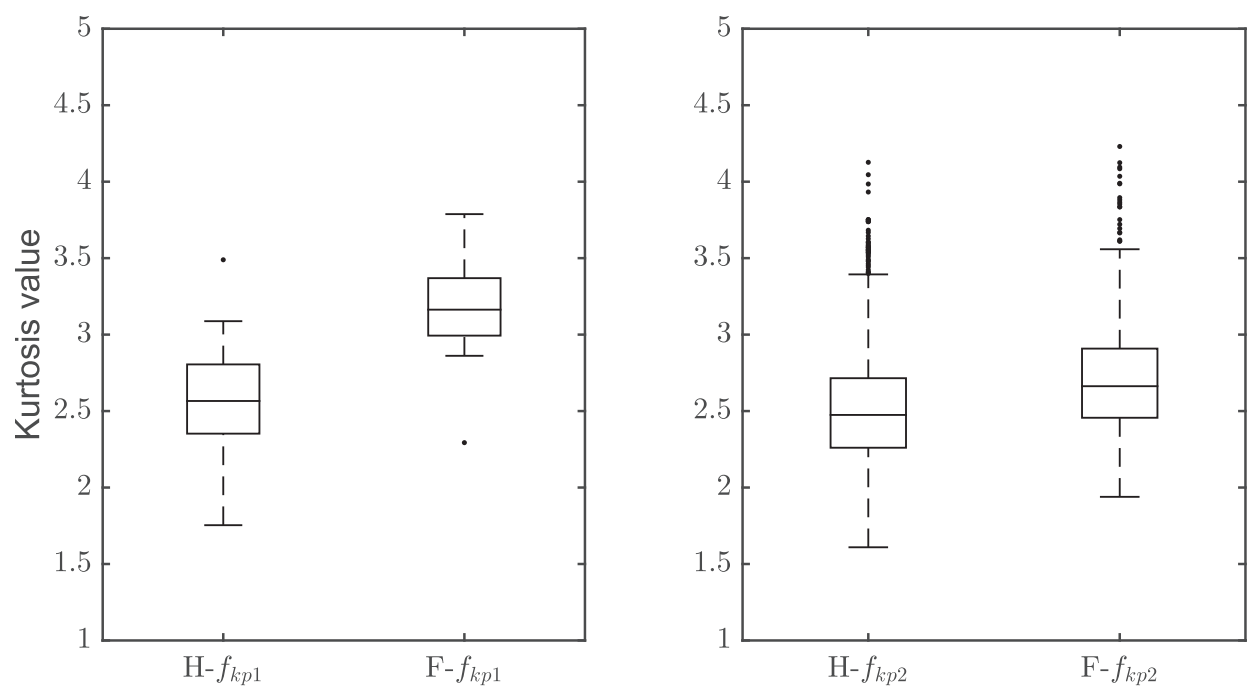

Fig. 18. Box plot showing the distribution of Kurtosis values of the residual for the healthy and faulty states of the gear, with measurements taken in all the bench conditions. Left: signal restricted to $4687.5 \mathrm{~Hz}$ sampling frequency, using the exact keyphasor times from the $93750 \mathrm{~Hz}$ reference. Right: signal restricted to $4687.5 \mathrm{~Hz}$ sampling frequency, using the modified keyphasor times with a jitter uncertainty of 0.4 cycles of the first gear mesh frequency.
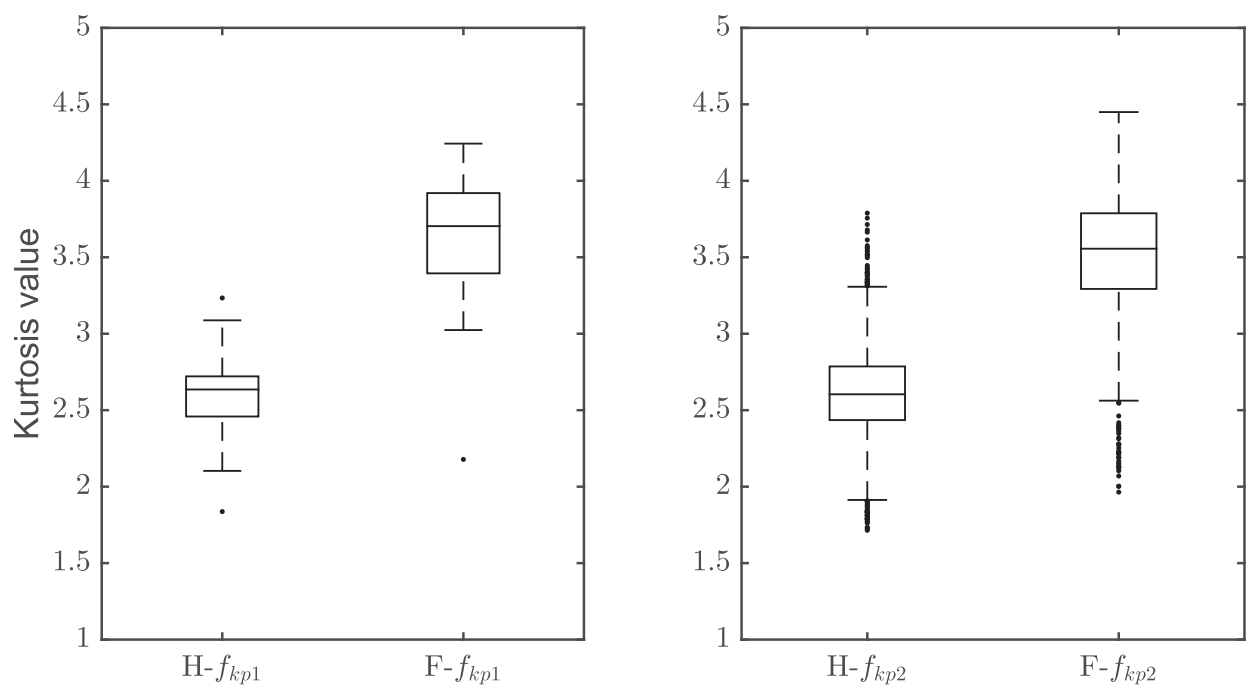

Fig. 19. Box plot showing the distribution of Kurtosis values of the residual for the healthy and faulty states of the gear, with measurements taken in all the bench conditions. Left: signal restricted to $9375 \mathrm{~Hz}$ sampling frequency, using the exact keyphasor times from the $93750 \mathrm{~Hz}$ reference. Right: signal restricted to $9375 \mathrm{~Hz}$ sampling frequency, using the modified keyphasor times with a jitter uncertainty of 0.2 cycles of the first gear mesh frequency.

\subsubsection{Data description}

The HUMS data used for this investigation was recorded within a period of one year from a fleet of fourteen Airbus type 2 helicopters performing different missions. The acquisitions were performed in various operating conditions, involving different regimes for the rotational speed of the rotor and for the transmitted torque from the engines. Main gearbox (MGB) and alternate gearbox (AGB) acquisitions comprise signals from seven accelerometers and two keyphasor signals. The two keyphasor signals provide respectively one pulse per revolution of the main rotor and of the tail drive shaft. Fig. 20 shows a schematics of the MGB and the two AGBs, indicating which components are assigned to which of the accelerometers. Kinematic ratios have been removed for proprietary reasons. Accelerometers are typically mounted on the gearbox case, close to the monitored components. Typically, acquisitions are divided in groups. Each acquisition group is launched when specific flight conditions are matched and consists of a synchronized acquisition from a set of sensors, performed with a configured sampling frequency for a configured duration. The available keyphasor signals are always sampled with the same sampling frequency of the accelerometer signals. As an example for this investigation, the amplitude of the first gear mesh frequency between the intermediate shaft and the combiner shaft is considered, measured from the MGBL accelerometer (see Fig. 20). 


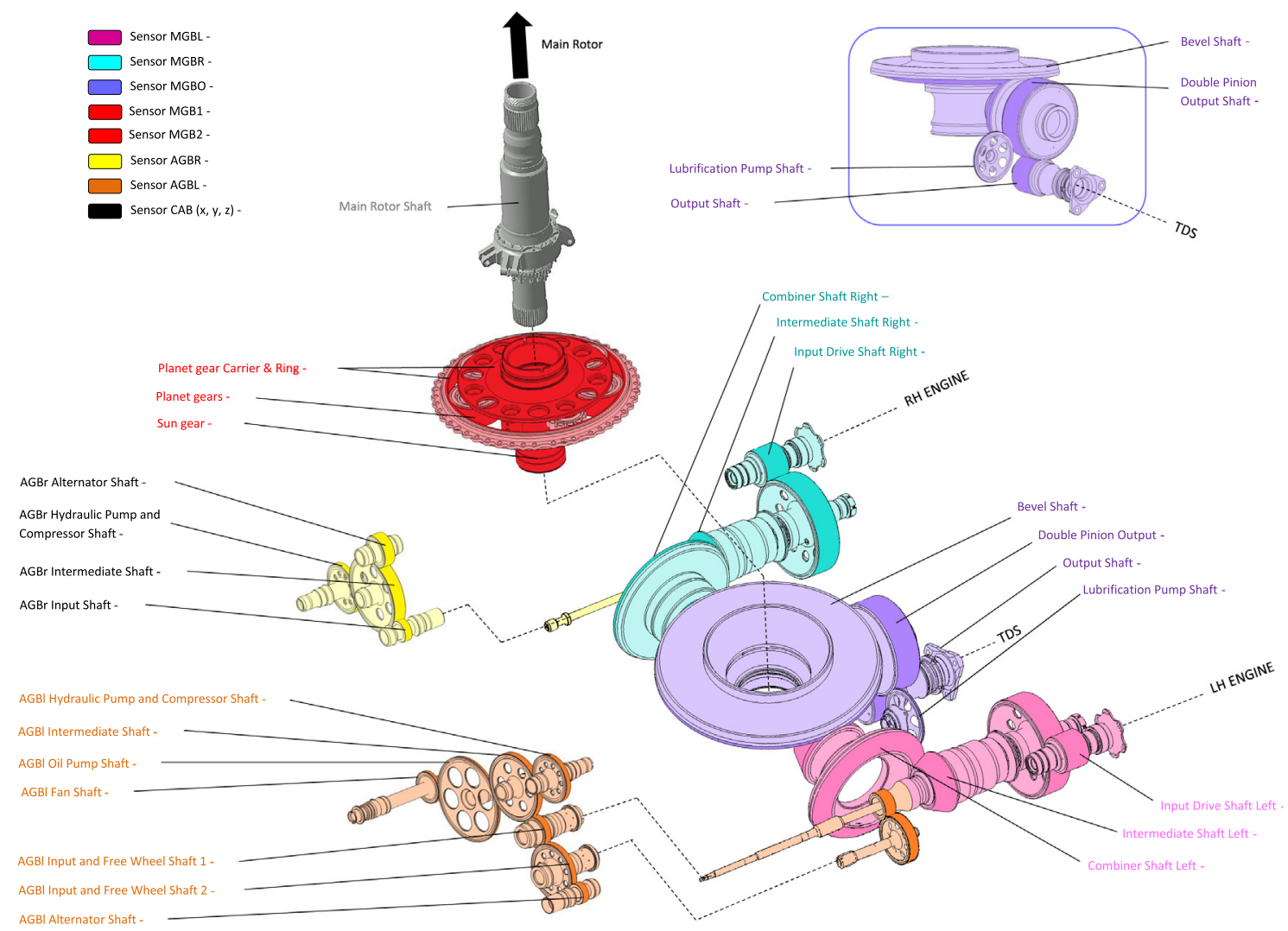

Fig. 20. Schematics of the type 2 gearbox and HUMS accelerometers.

Table 5

Parameters for order tracking and synchronous average using fleet data.

\begin{tabular}{lll}
\hline Parameter & Description [dimension] & Value \\
\hline$N_{t}$ & Number of revolutions of the reference shaft [-] & 21 \\
$r$ & Ratio of the target shaft speed to the reference shaft speed [-] & 5.8154 \\
$S P R$ & Angular samples per revolution [-] & 1024 \\
$\gamma_{1}$ & Order of the first gear mesh harmonic (in multiples of fundamental order) [-] & 23 \\
$N_{a}$ & Total number of acquisitions for the fleet [-] & 4546 \\
$\Delta$ & Artificially introduced pulse uncertainty [Fraction of gear mesh cycle] & 0.4
\end{tabular}

Such a choice is motivated again, as already explained in Section 5.1 for the type 1 test bench case, from the high signal to noise ratio and from the comparatively low frequency of this harmonic with respect to the maximum available sampling frequency for the vibration signal. The acquisition parameters and the kinematic parameters of interest for the present investigation are reported in Table 5, with the exception of the sampling frequency and the acquisition duration for proprietary reasons.

\subsubsection{Effect of keyphasor uncertainty in operational scenario}

With the objective of verifying that the results derived under assumptions (i)-(v) from Sections 2.1 and 3.2 hold in a general operating context, the following procedure is applied on each of the files from the HUMS flight data presented in Section 5.2.1:

1. The vector $x$ of vibration data from sensor MGBL is resampled synchronously with the intermediate shaft using pulse times from the tail rotor keyphasor signal, resulting in the angularly synchronous vector $x_{\theta}$;

2. The vector $x$ of vibration data from sensor MGBL is resampled synchronously with the intermediate shaft using pulse times from the tail rotor keyphasor signal modified with the addition of random variables from a uniform distribution $U_{[-\Delta / 2, \Delta / 2]}$, resulting in the angularly synchronous vector $x_{\hat{\theta}}$; 


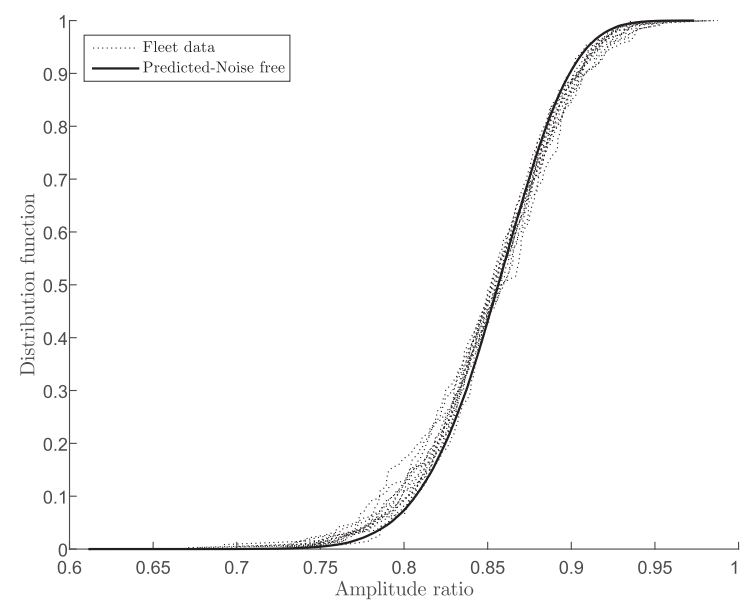

Fig. 21. Distribution function of the amplitude ratio parameter for the first gearmesh harmonic of the intermediate pinion. Black continous line: predicted; dotted lines: fleet data.

3. The synchronous average with the fundamental cycle equal to that of the intermediate input shaft is computed using all the available revolutions for both $x_{\theta}$ and $x_{\hat{\theta}}$;

4. The ratio of the amplitudes of the first gear mesh order from the synchronous average of $x_{\hat{\theta}}$ and the first gear mesh order from the synchronous average of $x_{\hat{\theta}}$ is stored.

5. The distribution functions of the obtained ratio values for each helicopter are compared to the theoretical predictions for the same ratio, obtained from Monte Carlo simulations using the developed model.

Since a keyphasor reference sampled with a lower sampling frequency was not available, differently from the case presented in Section 5.1, the uncertainty was simulated through the addition of the random values from a uniform distribution, which was already proved consistent in Section 5.1.2. Consistent results were obtained for different values of the uncertainty and considered harmonics (see Fig. 16). For $\Delta / T_{\text {mesh }}=0.4$, where $T_{\text {mesh }}$ is a meshing period of the intermediate pinion computed using the average rotational speed of the shaft during the record, results are shown in Fig. 21. Also for fleet data, Fig. 21 shows a sound agreement between the prediction and the experiment. Again, the higher dispersion of the fleet statistics can be explained from the effect of the noise, discussed in Section 3.3.1. The experiment confirms that the uncertainty from reference keyphasor pulses time of arrival is properly described by the modelling assumptions in an operational framework. The main effect of the uncertainty on the harmonic amplitude indicators lies in the introduced variance in the estimates, which shall be accounted for when considering the discriminability of two mechanical conditions based on, e.g., the estimated amplitude of some characteristic harmonics.

\section{Concluding remarks}

In this paper, a novel model for the random jittering error of the synchronization signal on the synchronous averaging procedure was introduced. A statistical analysis was performed to assess the distortion introduced by the error on the absolute value of the first order cyclostationary, or synchronous components in the signal, considering the dependency on the number of averages and on the entity of the uncertainty on the pulse times of arrival. The analysis considered uniform jitter variables in order to obtain practical approximated analytical solutions for the distribution of the estimated amplitudes, though the model lends itself to the introduction of any other random distributions for the jitter times. An analytical approximation was derived for the attenuation of a single harmonic, and the effect of additive noise was discussed. Extensive numerical simulations showed adequate performance of the developed model in predicting the statistics of the estimated synchronous harmonics, validating representativeness for the order tracking and synchronous average processes. The expressions derived for the analytical approximation provide means for quantifying the severity of random jitter effects under several combination of design parameters, also allowing to understand the effect of additive noise on the estimates and can, therefore, be used for optimize the configuration of industrial vibration diagnostics systems. It was found that for integer values of the ratio between the speed of the shaft of interest and the speed of the trigger shaft, the interaction due to jitter of synchronous, distinct orders is higher, leading to worse estimates. Using an experimental data set and an operational data set, the prediction capabilities of the model for the amplitude distribution of gear mesh harmonics were verified. Also, the effect of lowering the sampling frequency of the keyphasor signal on the accuracy of the estimates was assessed. This agrees with experimental investigations reported in the early order tracking literature, adding the consistency of a theoretical analysis and allowing the quantification of such effects. Further, it was shown that a piecewise linear approximation of the time-angle law is appropriate under nominally constant operating speed conditions and has the advantage of 
higher robustness to pulse times uncertainty. The results of this study can be employed in quantifying the effect of the pulse time uncertainty on the performance of those health monitoring indicators related to harmonic amplitudes, allowing to relate the estimation variance to the impact on the distinguishability of different mechanical states characterized by different values of the indicators. The attenuation effect is more relevant for less precise reference signals, both in terms of average attenuation and introduced scatter. As an additional remark, the developed general model for the synchronous averaging gives ground for extending the analysis to different cases of interest, e.g. it could be adapted for studying the uncertainty in tacholess synchronous averaging procedures.

\section{Appendix A. Approximation to the distribution of a linear combination of non-central chi-square random variables}

The algorithm reported below is a summary of the method developed in [47], and is used in the paper to derive an analytical approximation to the distribution of the synchronous components' amplitudes. The general expression for a quadratic form in the multivariate normal variable $\mathbf{X}$ is $Q=\mathbf{X}^{\top} \mathbf{A X}$. In the considered case $\mathbf{A}=\mathbf{I}$ and the procedure can therefore be summarized as follows:

1. Determine the eigenvalues $\lambda_{k}$ and the matrix of the normalized eigenvectors $\Lambda$ of $\Sigma$;

2. Compute $\Sigma^{-\frac{1}{2}}=\boldsymbol{\Lambda} \operatorname{diag}\left(\lambda_{k}^{-\frac{1}{2}}\right) \Lambda^{\top}$;

3. Compute $\mathbf{b}=\boldsymbol{\Lambda}^{\top} \boldsymbol{\Sigma}^{-\frac{1}{2}} E[\mathbf{g}]$;

4. Consider the transformed quadratic form $Q_{1}=\mathbf{W}_{\mathbf{1}}^{\top} \mathbf{A}_{\mathbf{1}} \mathbf{W}_{\mathbf{1}}$ defined as the quadratic form where the random normal variables have now identity covariance matrix and mean $\mathbf{b}$, and $\mathbf{A}_{\mathbf{1}}$ is defined as $\operatorname{diag}\left(\lambda_{k}\right)$;

5. Compute the $s$-th cumulant and the $h$-th moment of $\mathbf{Q}_{\mathbf{1}}$ respectively as:

$$
\begin{aligned}
& k(s)=2^{s-1} s !\left(\frac{\operatorname{tr}\left(\mathbf{A}_{\mathbf{1}}\right)^{s}}{s}+\mathbf{b}^{\top} \mathbf{A}_{\mathbf{1}}^{s} \mathbf{b}\right) \\
& \mu(h)=\sum_{i=0}^{h-1} \frac{(h-1) !}{(h-1-i) ! i !} k(h-i) \mu(i)
\end{aligned}
$$

6. With $X$ gamma-type random variable and $\mu(0)=1$, use Laguerre polynomial of degree $d$ to approximate the p.d.f. as:

$$
f_{X}(x)=\frac{x^{v} e^{-\frac{x}{\beta}}}{\beta^{v+1}} \sum_{j=0}^{d} \delta_{j}^{v} L_{j}^{v}\left(\frac{x}{\beta}\right)
$$

where:

$$
\begin{aligned}
& \delta_{j}^{v}=\sum_{k=0}^{j} \frac{(-1)^{k} j ! \mu_{Z}(j-k)}{k !(j-k) ! \Gamma(v+j-k+1)} \\
& L_{j}^{v}(x)=\sum_{k=0}^{j} d_{i, k}^{v} x^{k} \\
& d_{i, k}^{v}=\frac{(-1)^{i-k} \Gamma(i+v+1)}{(i-k) ! k ! \Gamma(v+k+1)} \\
& \beta=\frac{\mu_{X}(2)}{\mu_{X}(1)}-\mu_{X}(1) \\
& v=\frac{\mu_{X}^{2}(1)}{\mu_{X}(2)-\mu_{X}^{2}(1)}-1
\end{aligned}
$$

7. Finally, the cumulative distribution function (CDF) evaluated at $c>0$ is obtained by integration of Eq. (53) as:

$$
F_{X}(c)=\int_{0}^{c} f_{X}(x) d x=\sum_{i=0}^{d} \frac{\xi_{i}^{v}(\Gamma(v+i+1)-\Gamma(v+i+1, c / \beta))}{\Gamma(v+1)}
$$

in which the incomplete gamma function is denoted as:

$$
\Gamma(a, \theta)=\int_{\theta}^{\infty} t^{a-1} e^{-t} d t
$$

and: 


$$
\begin{gathered}
\xi_{k}^{v}= \begin{cases}1+\sum_{i=2}^{d} \eta_{i}^{v} d_{i, k}^{v}, & k=0 \\
\sum_{i=2}^{d} \eta_{i}^{v} d_{i, k}^{v}, & k=1 \\
\sum_{i=k}^{d} \eta_{i}^{v} d_{i, k}^{v}, & \text { otherwise }\end{cases} \\
\eta_{i}^{v}=\frac{i !}{\Gamma(v+i+1)} \sum_{k=0}^{i} d_{i, k}^{v} \mu_{X}(k)
\end{gathered}
$$

Eqs. (53) and (59) give an approximation to the probability density function and to the cumulative distribution function of the squared absolute value $\left|X_{S A}(\gamma)\right|^{2}$. Since $\left|X_{S A}(\gamma)\right|=\sqrt{\left|X_{S A}(\gamma)\right|^{2}}$ and the square root is an invertible and monotonically increasing function of the positive argument when negative solutions are not allowed, it also holds for the c.d.f.:

$$
F_{\left|X_{S A}(\gamma)\right|}(y)=P\left(\left|X_{S A}(\gamma)\right|^{2} \leqslant y^{2}\right)
$$

\section{References}

[1] C. Capdessus, M. Sidahmed, J.L. Lacoume, Cyclostationary processes: application in gear faults early diagnosis, Mech. Syst. Signal Process. 14 (3) (2000) 371-385.

[2] L. Bouillaut, M. Sidahmed, Cyclostationary approach and bilinear approach: comparison, applications to early diagnosis for helicopter gearbox and classification method based on HOCS, Mech. Syst. Signal Process. 15 (5) (2001) 923-943.

[3] A.C. McCormick, A.K. Nandi, Cyclostationarity in rotating machine vibrations, Mech. Syst. Signal Process. 12 (2) (1998) $225-242$.

[4] R.B. Randall, J. Antoni, S. Chobsaard, The relationship between spectral correlation and envelope analysis in the diagnostics of bearing faults and other cyclostationary machine signals, Mech. Syst. Signal Process. 15 (5) (2001) 945-962.

[5] J. Antoni, J. Daniere, F. Guillet, Effective vibration analysis of ic engines using cyclostationarity. part ia methodology for condition monitoring, J. Sound Vibr. 257 (5) (2002) 815-837.

[6] J. Antoni et al, Cyclostationary modelling of rotating machine vibration signals, Mech. Syst. Signal Process. 18 (6) (2004) $1285-1314$.

[7] L.J. Antolick et al., Evaluation of gear condition indicator performance on rotorcraft fleet, in: Proceedings of the 66th American Helicopter Society International Annual Forum, Phoenix, Arizona, U.S.A, 2010.

[8] G. D'Elia et al., Evolution of gear condition indicators for diagnostics of planetary gearboxes, in: The International Conference Surveillance 8. FRA, 2015.

[9] A. Raad, J. Antoni, M. Sidahmed, Indicators of cyclostationarity: theory and application to gear fault monitoring, Mech. Syst. Signal Process. 22 (3) (2008) 574-587.

[10] P. Več eř, M. Kreidl, R. Šamíd, Condition indicators for gearbox condition monitoring systems, Acta Polytech. 45 (6) (2005).

[11] R. Klein, Condition indicators for gears, in: Annual Conference of Prognostic and Health Management Society, 2012, pp. 1-8.

[12] V. Camerini, G. Coppotelli, S. Bendisch, Fault detection in operating helicopter drivetrain components based on support vector data description, Aerosp. Sci. Technol. 73 (2018) 48-60.

[13] F. Combet, L. Gelman, Optimal filtering of gear signals for early damage detection based on the spectral kurtosis, Mech. Syst. Signal Process. 23 (3) (2009) 652-668.

[14] H. Chandra, L. Gelman, Novel gear diagnosis technique based on spectral kurtosis, in: 23rd International Congress on Sound and Vibration, 2016.

[15] H. Li, Y. Zhang, H. Zheng, Angle domain average and CWT for fault detection of gear crack, in: Fifth International Conference on Fuzzy Systems and Knowledge Discovery, vol. 3, IEEE, Shandong (China), 2008, pp. 137-141.

[16] Z.S. Xiao et al., Application of time-domain average on fault diagnosis of planetary gear box, in: Hebei Gongye Daxue Xuebao/Journal of Hebei University of Technology (China) 32(6) (2003), pp. 99-102.

[17] Q. Leclere, N. Hamzaoui, Using the moving synchronous average to analyze fuzzy cyclostationary signals, Mech. Syst. Signal Process. 44 (1-2) (2014) 149-159.

[18] J.R. Ottewill, M. Orkisz, Condition monitoring of gearboxes using synchronously averaged electric motor signals, Mech. Syst. Signal Process. 38 (2) (2013) 482-498.

[19] N. Ahamed, Y. Pandya, A. Parey, Spur gear tooth root crack detection using time synchronous averaging under fluctuating speed, Measurement 52 (2014) 1-11.

[20] J. Zeidler et al, Adaptive enhancement of multiple sinusoids in uncorrelated noise, IEEE Trans. Acoust. Speech Signal Process. 26 (3) (1978) $240-254$.

[21] R.B. Randall, Y.J. Li, Diagnostics of planetary gear bearings in the presence of gear vibrations, in: Imeche Conference Transactions, vol. 5, Mechanical Engineering Publications, 1995, pp. 73-80.

[22] J. Antoni, R.B. Randall, Unsupervised noise cancellation for vibration signals: Part I-evaluation of adaptive algorithms, Mech. Syst. Signal Process. 18 (1) (2004) 89-101.

[23] J. Antoni, R.B. Randall, Unsupervised noise cancellation for vibration signals: Part II-a novel frequency-domain algorithm, Mech. Syst. Signal Process. 18 (1) (2004) 103-117.

[24] K.R. Fyfe, E.D.S. Munck, Analysis of computed order tracking, Mech. Syst. Signal Process. 11 (2) (1997) 187-205.

[25] F. Combet, L. Gelman, An automated methodology for performing time synchronous averaging of a gearbox signal without speed sensor, Mech. Syst. Signal Process. 21 (6) (2007) 2590-2606.

[26] F. Bonnardot et al, Use of the acceleration signal of a gearbox in order to perform angular resampling (with limited speed fluctuation), Mech. Syst. Signal Process. 19 (4) (2005) 766-785.

[27] M.D. Coats, R.B. Randall, Single and multi-stage phase demodulation based order-tracking, Mech. Syst. Signal Process. 44 (1-2) (2014) 86-117.

[28] D. Abboud et al, Deterministic-random separation in nonstationary regime, J. Sound Vibr. 362 (2016) 305-326.

[29] F. Bonnardot, Comparaison entre les analyses angulaire et temporelle des signaux vibratoires de machines tournantes. Etude du concept de cyclostationnarité floue, Institut National Polytechnique de Grenoble-INPG, 2004 (Ph.D. thesis).

[30] Q. Leclere, L. Pruvost, E. Parizet, Angular and temporal determinism of rotating machine signals: the diesel engine case, Mech. Syst. Signal Process. 24 (7) (2010) 2012-2020.

[31] H. Andre et al, Precision of the IAS monitoring system based on the elapsed time method in the spectral domain, Mech. Syst. Signal Process. 44 (1-2) (2014) 14-30.

[32] D. Remond, J. Antoni, R. Randall, Editorial for the special issue on Instantaneous Angular Speed (IAS) processing and angular applications, Mech. Syst. Signal Process. 1 (44) (2014) 1-4. 
[33] M. El Badaoui, F. Bonnardot, Impact of the non-uniform angular sampling on mechanical signals, Mech. Syst. Signal Process. 44 (1-2) (2014) 199-210.

[34] V. Camerini, G. Coppotelli, S. Bendisch, Experimental Evaluation and Statistical Analysis of Synchronous Averaging, Special Topics in Structural Dynamics, vol. 6, Springer, 2017, pp. 67-84.

[35] R. Potter, A new order tracking method for rotating machinery, Sound Vibr. 24 (9) (1990) 30-34.

[36] S. Braun, The extraction of periodic waveforms by time domain averaging, Acta Acustica United Acustica 32 (2) (1975) 69-77.

[37] P.D. McFadden, A revised model for the extraction of periodic waveforms by time domain averaging, Mech. Syst. Signal Process. 1 (1) (1987) 83-95.

[38] S. Braun, The synchronous (time domain) average revisited, Mech. Syst. Signal Process. 25 (4) (2011) 1087-1102.

[39] J. Antoni, Cyclostationarity by examples, Mech. Syst. Signal Process. 23 (4) (2009) 987-1036.

[40] D. Abboud et al, The spectral analysis of cyclo-non-stationary signals, Mech. Syst. Signal Process. 75 (2016) 280-300.

[41] L.F. Villa et al, Angular resampling for vibration analysis in wind turbines under non-linear speed fluctuation, Mech. Syst. Signal Process. 25 (6) (2011) $2157-2168$.

[42] P.D. McFadden, Interpolation techniques for the time domain averaging of vibration data with application to helicopter gearbox monitoring. Tech. rep., Aeronaut. Res. Labs Melbourne (Australia) (1986).

[43] D. Abboud, Generalization of the synchronous average for Deterministic/Random Separation under speed varying conditions, in: VISHNO $2014,2014$.

[44] O. Meste, H. Rix, Jitter statistics estimation in alignment processes, Signal Process. 51 (1) (1996) 41-53.

[45] P. Borghesani et al, Order tracking for discrete-random separation in variable speed conditions, Mech. Syst. Signal Process. 30 (2012) 1-22.

[46] D. Vats, J.M. Flegal, G.L. Jones, Multivariate output analysis for Markov chain Monte Carlo (2019). http://dx.doi.org/10.1093/biomet/asz002.

[47] H.T. Ha, Serge B. Provost, An accurate approximation to the distribution of a linear combination of non-central chi-square random variables, Revstat Stat. J 11 (3) (2013) 231-254.

[48] P.D. McFadden, Examination of a technique for the early detection of failure in gears by signal processing of the time domain average of the meshing vibration, Mech. Syst. Signal Process. 1 (2) (1987) 173-183.

[49] G. Dalpiaz, A. Rivola, R. Rubini, Effectiveness and sensitivity of vibration processing techniques for local fault detection in gears, Mech. Syst. Signal Process. 14 (3) (2000) 387-412.

[50] P.D. McFadden, Detecting fatigue cracks in gears by amplitude and phase demodulation of the meshing vibration, J. Vibr., Acoust., Stress Reliab. Des. 108 (2) (1986) 165-170.

[51] W. Wang, A.K. Wong, Autoregressive model-based gear fault diagnosis, J. Vibr. Acoust. 124 (2) (2002) 172-179.

[52] N. Sawalhi, R.B. Randall, H. Endo, The enhancement of fault detection and diagnosis in rolling element bearings using minimum entropy deconvolution combined with spectral kurtosis, Mech. Syst. Signal Process. 21 (6) (2007) 2616-2633.

[53] G.L. McDonald, Q. Zhao, M.J. Zuo, Maximum correlated Kurtosis deconvolution and application on gear tooth chip fault detection, Mech. Syst. Signal Process. 33 (2012) 237-255. 